\title{
Crystal field excitations and magnons: Their roles in oxyselenides $\operatorname{Pr}_{2} \mathrm{O}_{2} M_{2} \mathrm{OSe}_{2}(M=\mathrm{Mn}, \mathrm{Fe})$
}

\author{
R. K. Oogarah, ${ }^{1}$ C. P. J. Stockdale, ${ }^{2}$ C. Stock,${ }^{2}$ J. S. O. Evans, ${ }^{3}$ A. S. Wills,${ }^{4}$ J. W. Taylor, ${ }^{5}$ and E. E. McCabe ${ }^{1, *}$ \\ ${ }^{1}$ School of Physical Sciences, Ingram Building, University of Kent, Canterbury, Kent, United Kingdom \\ ${ }^{2}$ School of Physics and Astronomy, University of Edinburgh, Edinburgh, EH9 3JZ, United Kingdom \\ ${ }^{3}$ Department of Chemistry, Durham University, Lower Mountjoy, South Road, Durham, DH1 3LE, United Kingdom \\ ${ }^{4}$ Department of Chemistry, University College London, 20 Gordon Street, London, WC1H OAJ, United Kingdom \\ ${ }^{5}$ ISIS Facility, Rutherford Appleton Laboratory, Chilton, Didcot, OX11 0QX, United Kingdom \\ (Received 25 October 2016; revised manuscript received 31 March 2017; published 30 May 2017)
}

\begin{abstract}
We present the results of neutron scattering experiments to study the crystal and magnetic structures of the Mottinsulating transition metal oxyselenides $\operatorname{Pr}_{2} \mathrm{O}_{2} M_{2} \mathrm{OSe}_{2}(M=\mathrm{Mn}, \mathrm{Fe})$. The structural role of the non-Kramers $\mathrm{Pr}^{3+}$ ion was investigated, and analysis of $\mathrm{Pr}^{3+}$ crystal field excitations was performed. Long-range order of $\mathrm{Pr}^{3+}$ moments in $\mathrm{Pr}_{2} \mathrm{O}_{2} \mathrm{Fe}_{2} \mathrm{OSe}_{2}$ can be induced by an applied magnetic field.
\end{abstract}

DOI: 10.1103/PhysRevB.95.174441

\section{INTRODUCTION}

Much research is directed towards understanding the parent phases of unconventional superconductors and, in particular, their structures, exchange interactions, and the driving forces for structural distortions. The phase diagram for cuprate superconductors derives from parent Mott-insulating antiferromagnetic (AFM) phases and unconventional superconductivity is induced by charge doping [1-3]. The iron-based superconducting systems are less clearly understood [4-6], with poorly metallic or semimetallic parent phases, raising the question as to whether a localized or an itinerant/spin-density wave model is more appropriate [7].

The " $M_{2} \mathrm{O}$ " oxyselenides [e.g., $\mathrm{La}_{2} \mathrm{O}_{2} M_{2} \mathrm{OSe}_{2}(M=$ $\mathrm{Mn}, \mathrm{Fe}, \mathrm{Co})]$ adopt tetragonal crystal structures composed of alternating $\left[\mathrm{La}_{2} \mathrm{O}_{2}\right]^{2+}$ fluorite like and $\left[\mathrm{M}_{2} \mathrm{O}\right]^{2+}$ layers separated by selenide anions [Fig. 1(a)]. This gives an unusual $\mathrm{M}^{2+}$ coordination environment with pseudo-octahedral coordination by two oxide anions (in-plane) and four selenide anions (above and below the $\left[\mathrm{M}_{2} \mathrm{O}\right]^{2+}$ sheets) [Fig. 1(b)] [8]. Electronic structure calculations on $\mathrm{La}_{2} \mathrm{O}_{2} \mathrm{Fe}_{2} \mathrm{OSe}_{2}$ reveal relatively narrow $\mathrm{Fe}^{2+} 3 d$ bands in this layered material, pointing to significant correlation effects [9]. This is consistent with electrical resistivity measurements [9-11] and suggests that these " $\mathrm{Fe}_{2} \mathrm{O}$ " materials provide a suitable Mott-insulating reference system in which to study the exchange interactions and anisotropy in layered mixed-anion phases [12].

The $\mathrm{La}_{2} \mathrm{O}_{2} M_{2} \mathrm{OSe}_{2}(M=\mathrm{Mn}, \mathrm{Fe}, \mathrm{Co})$ oxyselenides order antiferromagnetically on cooling $\left(T_{\mathrm{N}}=160-168 \mathrm{~K}, 89 \mathrm{~K}\right.$, and $220 \mathrm{~K}$ for Mn [13-15], Fe [16,17], and Co [18-20] analogues, respectively). There are three magnetic exchange interactions in the $M_{2} \mathrm{O}$ layers: nearest-neighbor (nn) AFM exchange $J_{1}$, next-nearest-neighbor (nnn) $180^{\circ} M-\mathrm{O}-M$ AFM exchange $J_{2^{\prime}}$ and nnn $\sim 97^{\circ} M$-Se- $M$ exchange $J_{2}$ [Fig. 1(c)]. The relative strengths of these exchange interactions vary with $M$ electronegativity: for $M=\mathrm{Mn}, \mathrm{nn} J_{1}$ interactions dominate, giving $G$-type AFM order with moments perpendicular to the $\left[\mathrm{M}_{2} \mathrm{O}\right]^{2+}$ layers [Fig. 1(d)] $[14,15]$, while for the more electronegative $M=\mathrm{Co}^{2+}$ analogue, $180^{\circ} J_{2^{\prime}}$ interactions

\footnotetext{
*e.e.mccabe@kent.ac.uk
}

dominate, leading to a magnetic structure with $k$ vector $k=(1 / 21 / 20)$ with in-plane moments and $n n$ moments perpendicular to one another $[18,19,21,22]$. For $M=\mathrm{Fe}, \mathrm{Fe}-\mathrm{Se}-\mathrm{Fe}$ $J_{2}$ interactions are ferromagnetic (FM) [10] (consistent with the FM chains of edge-linked $\mathrm{FeSe}_{4}$ tetrahedra in $\mathrm{Ce}_{2} \mathrm{O}_{2} \mathrm{FeSe}_{2}$ ) $[23,24] . \mathrm{La}_{2} \mathrm{O}_{2} \mathrm{Fe}_{2} \mathrm{OSe}_{2}$ adopts a two- $k$-vector magnetic structure $\left[k=\left(1 / 20^{1 / 2}\right)\right.$ and $\left.k=\left(0^{1 / 2} 1 / 2\right)\right]$ with in-plane moments directed along the Fe-O bonds, with FM $J_{2}$ and AFM $J_{2^{\prime}}$ interactions consistent with theory and nearest-neighbor moments orthogonal to one another [Fig. 1(e)] (referred to here as the $2 k$ structure) $[11,12,17,25]$. Second-order exchange interactions alone are insufficient to stabilize this $2 k$ magnetic structure and higher order terms (e.g., from $\mathrm{Fe}^{2+}$ spin anisotropy) are needed. The onset of AFM order is two-dimensional (2D)-Ising-like [12], and inelastic neutron scattering (INS) experiments revealed an anisotropy gap $(\sim 5 \mathrm{meV}$ at $2 \mathrm{~K})$ and very weak magnetic exchange interactions [17], consistent with band narrowing reported for these materials [9].

Lanthanide magnetism can also influence the magnetism of the transition metal sublattice, with $\mathrm{Fe}^{2+}$ spin reorientation driven by lanthanide magnetic order in 1111 materials $\mathrm{PrFeAsO}$ and $\mathrm{NdFeAsO}[26,27]$. In the layered copper oxide superconducting systems, the electronic state of $\mathrm{Pr}^{3+}$ ions (leading to magnetic or nonmagnetic ground states) was key to whether copper-based superconductivity evolved on cooling [28-30]. This has prompted significant research into possible coupling between the lanthanide and transition metal sublattices in layered systems [24,31-45]. Probing the crystalline electric field (CEFs) of the lanthanide ions by INS experiments has given insights into both the lanthanide magnetism and the transition metal sublattice $[41,46]$. In addition, the non-Kramers $4 f^{2}\left({ }^{3} \mathrm{H}_{4}\right) \mathrm{Pr}^{3+}$ ion is also susceptible to symmetry-lowering structural distortions driven by $4 f$ electron degrees of freedom [32]. In PrMnAsO and PrMnSbO, structural distortions occur at $\sim 35 \mathrm{~K}$, which lower the symmetry of the $\mathrm{Pr}^{3+}$ site from $4 \mathrm{~mm}$ to $\mathrm{mm} 2$, accompanied by long-range order of $\mathrm{Pr}^{3+}$ moments and reorientation of $\mathrm{Mn}^{2+}$ moments [32,33]. An analogous structural distortion occurs in $\operatorname{Pr}_{2} \mathrm{O}_{2} \mathrm{Mn}_{2} \mathrm{OSe}_{2}$ below $36 \mathrm{~K}$, lowering the crystal symmetry from I4/mmm to Immm, but, unlike PrMnAsO and PrMnSbO, there is no evidence for long-range ordering of $\mathrm{Pr}^{3+}$ moments in $\mathrm{Pr}_{2} \mathrm{O}_{2} \mathrm{Mn}_{2} \mathrm{OSe}_{2}$. 


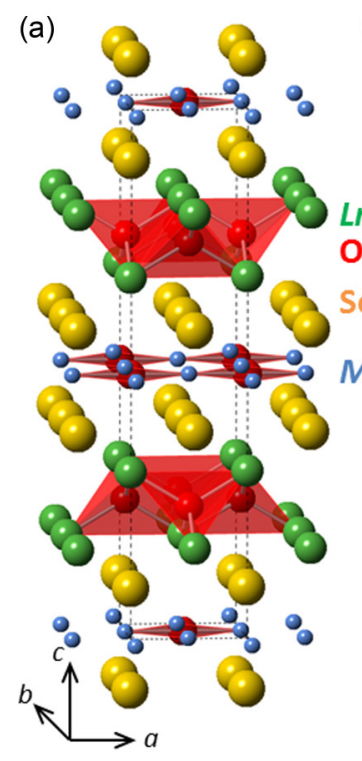

(b)

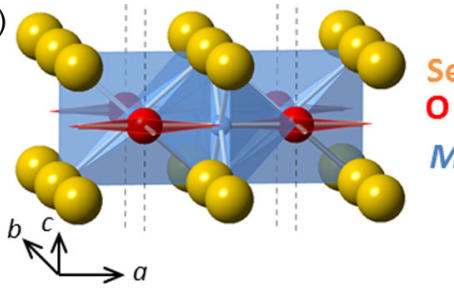

$J_{2}:$ nnn exchange $\left(\sim 97^{\circ} M-\mathrm{Se}-M\right)$

$J_{2^{\prime}}:$ nnn exchange $\left(180^{\circ} \mathrm{M}-\mathrm{O}-\mathrm{M}\right)$

$J_{1}: \mathrm{nn}$ exchange (direct $/ 90^{\circ} \mathrm{M}-\mathrm{O}$

(d)

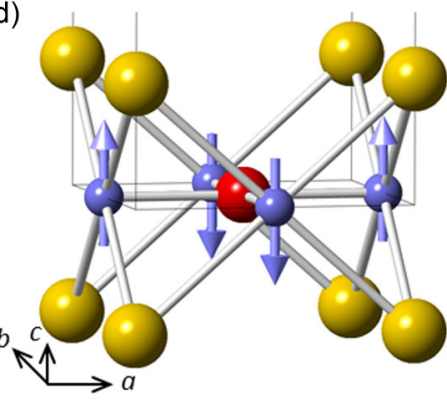

(c)
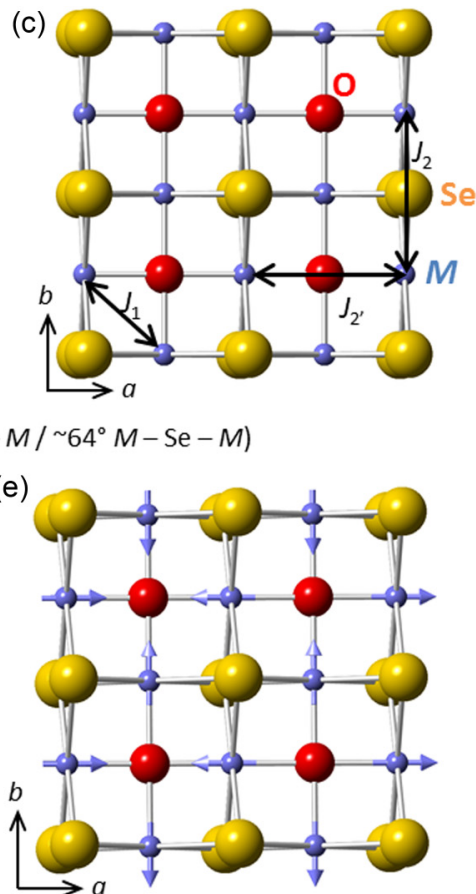

FIG. 1. Structure of $L n_{2} \mathrm{O}_{2} M_{2} \mathrm{OSe}_{2}$ showing (a) tetragonal unit cell, (b) pseudo-octahedral $M \mathrm{Se}_{4} \mathrm{O}_{2}$ coordination polyhedra, and (c) $M$-Se-O layers with exchange interactions labeled; (d) in-plane magnetic order in $\mathrm{Pr}_{2} \mathrm{O}_{2} \mathrm{Mn}_{2} \mathrm{OSe}_{2}$ [14] and (e) $2 k$ in-plane magnetic order in $\mathrm{Pr}_{2} \mathrm{O}_{2} \mathrm{Fe}_{2} \mathrm{OSe}_{2}$, with $M^{2+}$ moments shown by blue arrows. $L n, M(M=\mathrm{Mn}, \mathrm{Fe}), \mathrm{O}$, and Se ions are shown in green, blue, red, and yellow, respectively.

In this paper we investigate the structural changes in $\mathrm{Pr}_{2} \mathrm{O}_{2} \mathrm{Fe}_{2} \mathrm{OSe}_{2}$ on cooling and observe a subtle orthorhombic structural distortion at $\sim 23 \mathrm{~K}$. We compare the lowtemperature neutron powder diffraction (NPD) data collected in 0 and $5 \mathrm{~T}$ applied fields to study the metamagnetic phase transition in this material. Using INS, we investigate the magnetism in $\mathrm{Pr}_{2} \mathrm{O}_{2} \mathrm{Mn}_{2} \mathrm{OSe}_{2}$ and confirm the lack of an anisotropy gap for this $\mathrm{Mn}^{2+}$ material, in contrast to $\mathrm{Fe}^{2+}$ analogues [17]. We also investigate the local electronic crystalline electric field environment by studying the low-energy CEFs of the $\mathrm{Pr}^{3+}$ ion for both $(M=\mathrm{Mn}, \mathrm{Fe})$ materials. We show how the transition metal magnetic excitations might couple with the $\mathrm{Pr}^{3+} \mathrm{CEFs}$ and influence the structural behavior.

\section{EXPERIMENTAL DETAILS}

Five grams of $\mathrm{Pr}_{2} \mathrm{O}_{2} \mathrm{Fe}_{2} \mathrm{OSe}_{2}$ were prepared by the solid state reaction of $\mathrm{Pr}_{2} \mathrm{O}_{3}$ [prepared by heating $\operatorname{Pr}_{6} \mathrm{O}_{11}$ powder (Alfa Aesar, 99.99\%) in a flow of $5 \% \mathrm{H}_{2}(\mathrm{~g}) / 95 \% \mathrm{~N}_{2}(\mathrm{~g})$ to $1000^{\circ} \mathrm{C}$ and holding at this temperature for $10 \mathrm{~h}$, before furnace cooling in the same gas flow to room temperature), $\mathrm{Fe}$ (Aldrich, 99.9\%), and Se (Alfa Aesar, 99.999\%). Stoichiometric quantities of these reagents were intimately ground together by hand using an agate pestle and mortar. The resulting grey powder was pressed into several 5-mm-diameter pellets using a uniaxial press. These pellets were slowly heated in an evacuated, sealed quartz tube to $600^{\circ} \mathrm{C}$ and held at this temperature for $12 \mathrm{~h}$, and then heated to $1000^{\circ} \mathrm{C}$ and held for $12 \mathrm{~h}$. The sample was then cooled to room temperature in the furnace. $\mathrm{Pr}_{2} \mathrm{O}_{2} \mathrm{Mn}_{2} \mathrm{OSe}_{2}$ was prepared as described elsewhere [14]. Preliminary structural characterization was carried out using powder x-ray diffraction data collected on a Bruker D8 Advance diffractometer from $5^{\circ}$ to $100^{\circ} 2 \theta$. The diffractometer was fitted with a LynxEye silicon strip detector (step size $0.021^{\circ}$ ) and an Oxford Cryosystems PheniX closedcycle refrigerator (CCR) cryostat to access temperatures between $12 \mathrm{~K}$ and $300 \mathrm{~K}$. The NPD data were collected for $\mathrm{Pr}_{2} \mathrm{O}_{2} \mathrm{Fe}_{2} \mathrm{OSe}_{2}$ on the time-of-flight (TOF) diffractometer Wish on target station 2 at the ISIS spallation neutron source. For scans collected in zero applied field, the powder was placed in a 6-mm-diameter cylindrical vanadium can (to a height of $\sim 6 \mathrm{~cm})$. A $25 \mathrm{~min}(15 \mu \mathrm{A} \mathrm{h})$ scan was carried out at $1.5 \mathrm{~K}$, then $15 \mathrm{~min}(10 \mu \mathrm{A} \mathrm{h})$ scans were collected on warming at $\sim 1.5 \mathrm{~K}$ intervals up to $100 \mathrm{~K}$, followed by $8 \min (5 \mu \mathrm{A} \mathrm{h})$ scans at $10 \mathrm{~K}$ intervals up to $150 \mathrm{~K}$. Data were also collected in applied magnetic fields up to $5 \mathrm{~T}$ by placing the pelletized sample in a $6 \mathrm{~mm}$ vanadium can and securing it. Twenty-five minute (16 $\mu \mathrm{A} \mathrm{h}$ ) scans were collected every $4 \mathrm{~K}$ on warming from $2 \mathrm{~K}$ to $110 \mathrm{~K}$ in a $5 \mathrm{~T}$ applied magnetic field, and then $19 \mathrm{~min}$ $(12.5 \mu \mathrm{A} \mathrm{h})$ scans were collected at $2 \mathrm{~K}$ in increments of $0.5 \mathrm{~T}$ up to $4.5 \mathrm{~T}$. Rietveld refinements [47] were performed using TOPAS-Academic software [48]. Sequential refinements were carried out using data collected between $1.5 \mathrm{~K}$ and $150 \mathrm{~K}$ using TOPAS-Academic and local subroutines. The high resolution bank of data (bank 5, 10.5-85.0 ms, 1-4 ) was used to investigate structural changes as a function of temperature, whilst the higher $d$-spacing bank 2 data (10.5-85.0 ms, 1-8 $\AA$ ) were used to study the magnetic behavior of $\mathrm{Pr}_{2} \mathrm{O}_{2} \mathrm{Fe}_{2} \mathrm{OSe}_{2}$. For sequential refinements, background (shifted Chebyshev polynomial, 12 terms) unit cell parameters for the nuclear phase, as well as atomic coordinates, thermal displacement parameters, a peak shape for each phase, and the $\mathrm{Fe}^{2+}$ magnetic 
(a)

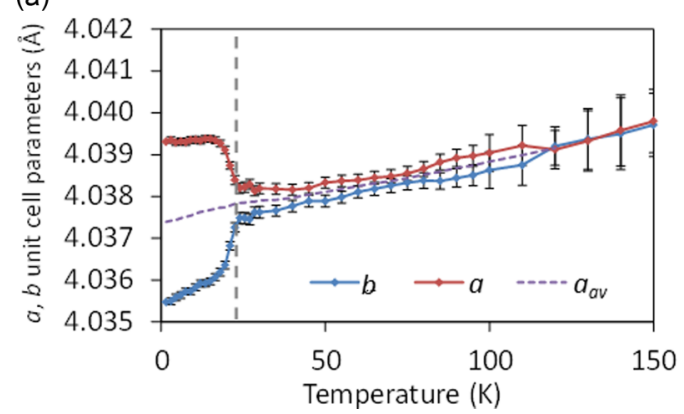

(b)

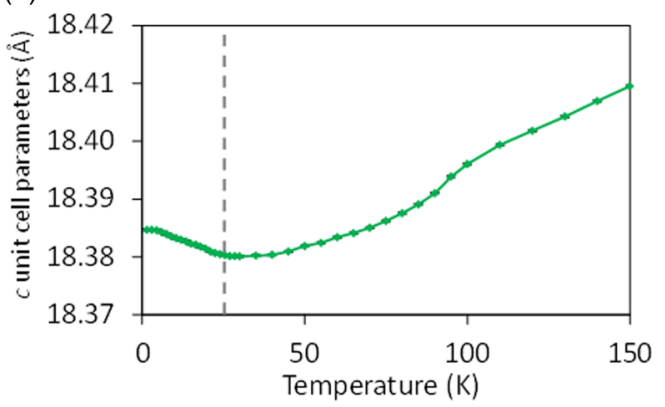

FIG. 2. Unit cell parameters from sequential NPD Rietveld refinements using the Immm model. Dashed grey line is at $23 \mathrm{~K}$, and lattice parameters $a$ and $b$ are shown in red and blue, respectively, with the average in-plane lattice parameter, $a_{\mathrm{a} v}$, shown by dashed purple line.

moment were refined. For data collected in an applied magnetic field, three relatively strong reflections [022 at $29600 \mu \mathrm{s}(d=$ $1.42 \AA), 311$ at $25200 \mu \mathrm{s}(d=1.21 \AA)$, and 042 at $188700 \mu \mathrm{s}$ ( $d=0.90 \AA$ )] due to aluminum (from the sample environment) were observed in the high resolution data bank, and these were Pawley-fitted with a face-centered-cubic model.

For INS measurements, the samples were packed into $\mathrm{Al}$ foil envelopes and placed in Al cans. Magnetic excitations were measured using the MARI direct geometry chopper spectrometer at the ISIS source. Incident beam energies $E_{i}=10,40$, or $85 \mathrm{meV}$ were selected using a Gd Fermi chopper set at frequencies of 250,150 , or $250 \mathrm{~Hz}$, respectively. A $t_{0}$ chopper (spinning at $50 \mathrm{~Hz}$ ) was used to block fast neutrons, and a thick disk chopper (also spinning at $50 \mathrm{~Hz}$ ) was used to improve the background from neutrons above the Gd absorption edge. The simulated spectra shown were obtained by convoluting the experimental resolution (measured with a vanadium standard) with a Lorentzian lineshape with terms on the energy gain and loss sides to ensure detailed balance [49]. SpinW, which uses linear spin wave theory to simulate inelastic neutron scattering on magnetic materials [50] by solving the Heisenberg Hamiltonian, was used here to simulate INS spectra for various magnetic models (further details are given below).

\section{RESULTS AND DISCUSSION}

\section{A. Low-temperature structural behavior for $\operatorname{Pr}_{2} \mathrm{O}_{2} \mathrm{Fe}_{2} \mathrm{OSe}_{2}$}

NPD data collected for $\operatorname{Pr}_{2} \mathrm{O}_{2} \mathrm{Fe}_{2} \mathrm{OSe}_{2}$ could be fitted by the tetragonal structure reported by $\mathrm{Ni}$ et al. [36] To check our sample composition, data collected at $110 \mathrm{~K}$ (above $T_{\mathrm{N}}$ ) were fitted with this model with a global temperature factor and allowing site occupancies to refine (the $\operatorname{Pr}^{3+}$ site occupancy was fixed at unity). This gave occupancies within an estimated standard deviation (esd) of unity for Se, O(1) and $\mathrm{O}(2)$ sites, and an occupancy of $0.965(7)$ for the Fe site. This is very close to the ideal composition and implies formal oxidation states very close to $\mathrm{Pr}^{3+}, \mathrm{Fe}^{2+}, \mathrm{O}^{2-}$, and $\mathrm{Se}^{2-}$. The slight $\mathrm{Fe}$ deficiency may imply some oxidation of $\mathrm{Fe}^{2+}$ and $\operatorname{Pr}^{3+}$ ions, although any oxidation must be very small given the good agreement in room temperature lattice parameters ( $a=4.04470(5) \AA, c=18.4475(3) \AA$ ) for that expected for this series of $\mathrm{Ln}_{2} \mathrm{O}_{2} \mathrm{Fe}_{2} \mathrm{OSe}_{2}$ materials [37].

Sequential Rietveld refinements in zero field showed that the unit cell parameters decreased on cooling, similar to behavior reported for other $L n_{2} \mathrm{O}_{2} \mathrm{Fe}_{2} \mathrm{OSe}_{2}$ analogues, with a more rapid decrease in the $c$ parameter below $T_{\mathrm{N}}$, presumably due to magnetostrictive effects [17,37]. However, a slight increase in the $c$ parameter was observed below $\sim 22.5 \mathrm{~K}$ (see Fig. 2(b) and Supplemental Material [51]). Data at $2 \mathrm{~K}$ could be fitted by a nuclear phase of $14 / \mathrm{mmm}$ symmetry and a magnetic phase (see Supplemental Material [51]) analogous to that described for $\mathrm{La}_{2} \mathrm{O}_{2} \mathrm{Fe}_{2} \mathrm{OSe}_{2}$ [17].

Although no additional peaks and no clear splitting of diffraction peaks were observed at low temperature, we note that there is a marked increase in the $d$-spacing dependent peak shape term (which describes strain broadening) below $\sim 22.5 \mathrm{~K}$. Sequential refinements (using only the high resolution $153^{\circ}$ bank data) with a Pawley phase of $I 4 / \mathrm{mmm}$ symmetry indicated that this broadening is anisotropic, affecting $h 00 / 0 k 0$ reflections much more than hhl reflections (Supplemental Material [51]). Allowing the nuclear phase to undergo an orthorhombic distortion gives stable refinements consistent with a subtle distortion to Immm symmetry $(a=4.0390(2) \AA, b=4.0349(1) \AA, c=$ $18.3820(8) \AA$, volume $=299.56(2) \AA^{3}$ at $\left.1.5 \mathrm{~K}\right)$ involving displacement of $\mathrm{O}(2)$ along [001] away from the ideal $4 d$ site in I4/mmm $(z=0.75)$ (Fig. 2 and Supplemental Material [51]). NPD data collected in a $5 \mathrm{~T}$ applied magnetic field show that $\mathrm{Pr}_{2} \mathrm{O}_{2} \mathrm{Fe}_{2} \mathrm{OSe}_{2}$ has similar structural behavior with and without an applied magnetic field (Supplemental Material [51]).

With no clear peak splitting, it is hard to be definitive, but our analysis suggests that below $\sim 23 \mathrm{~K}, \mathrm{Pr}_{2} \mathrm{O}_{2} \mathrm{Fe}_{2} \mathrm{OSe}_{2}$ undergoes a distortion similar to that observed for $\mathrm{Pr}_{2} \mathrm{O}_{2} \mathrm{Mn}_{2} \mathrm{OSe}_{2}$ [14] but which is more subtle for the $\mathrm{Fe}$ analogue [orthorhombicity, defined as $2(a-b) /(a+b)$, is $2.0 \times 10^{-3}$ for $\mathrm{Pr}_{2} \mathrm{O}_{2} \mathrm{Mn}_{2} \mathrm{OSe}_{2}$ at $12 \mathrm{~K}$ [14], compared with $1.0 \times 10^{-3}$ for $\mathrm{Pr}_{2} \mathrm{O}_{2} \mathrm{Fe}_{2} \mathrm{OSe}_{2}$ at $\left.1.5 \mathrm{~K}(0 \mathrm{~T})\right]$. This structural distortion may be the origin of the peak in heat capacity reported by $\mathrm{Ni}$ et al. for $\mathrm{Pr}_{2} \mathrm{O}_{2} \mathrm{Fe}_{2} \mathrm{OSe}_{2}$ at about $23 \mathrm{~K}$ [36]. Analogous distortions are observed in other materials containing fluorite like $\left[\mathrm{Pr}_{2} \mathrm{O}_{2}\right]^{2+}$ layers, including PrMnSbO [32] (orthorhombicity $4.3 \times$ $10^{-3}$ at $4 \mathrm{~K}$ ) and $\mathrm{PrMnAsO}_{0.95} \mathrm{~F}_{0.05}$ [33] (orthorhombicity $0.8 \times 10^{-3}$ at $\left.10 \mathrm{~K}\right)$. These distortions, ascribed to the $\operatorname{Pr}^{3+} 4 f^{2}$ orbital degrees of freedom [32], lower the $\operatorname{Pr}^{3+}$ site symmetry ( $4 \mathrm{~mm}$ to $2 \mathrm{~mm}$ ) as oxide ions are displaced along [001], giving two long and two short Pr-O bonds, accompanied by loss of the $C_{4}$ rotational symmetry and the slight increase in the $c$ lattice parameter. In both PrMnSbO [32] and $\mathrm{PrMnAsO}_{0.95} \mathrm{~F}_{0.05}$ [33], this distortion is accompanied by long-range ordering of $\mathrm{Pr}^{3+}$ magnetic moments $\left(T_{\mathrm{N}, \mathrm{Pr}} \approx 80 \mathrm{~K}\right.$ 

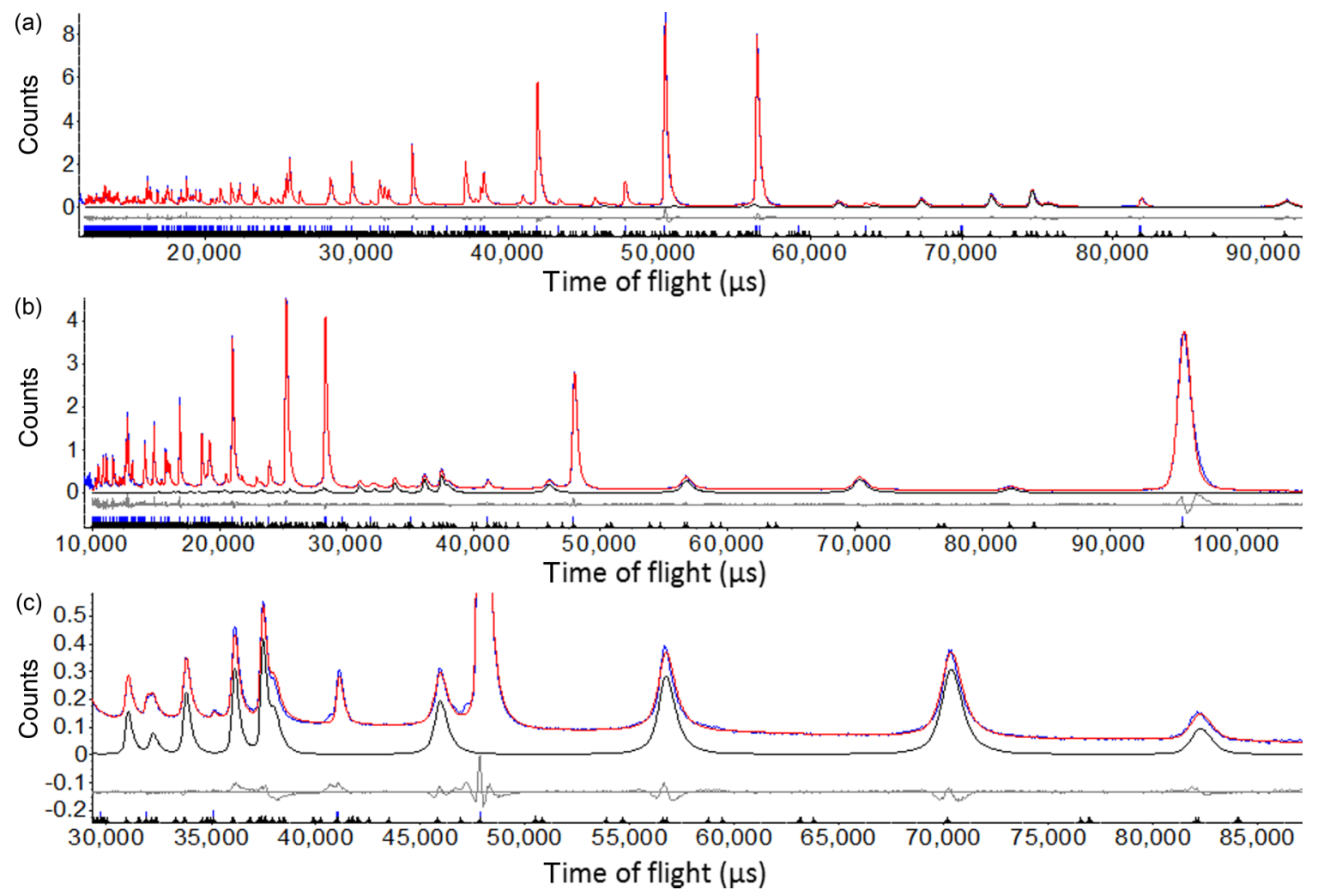

FIG. 3. Rietveld refinement profiles from combined refinement using (a) $153^{\circ}$ bank data $\left(\sim 0.6-4.5 \AA d\right.$-spacing range) and (b) $59^{\circ}$ bank data ( 1-10 $\AA$-spacing range) collected for $\mathrm{Pr}_{2} \mathrm{O}_{2} \mathrm{Fe}_{2} \mathrm{OSe}_{2}$ at $1.5 \mathrm{~K}$; (c) highlights are the higher $d$-spacing region of $59^{\circ}$ bank data emphasizing the magnetic reflections. Observed and calculated (upper) and difference profiles are shown by blue points and red and gray lines, respectively. Magnetic intensity is highlighted by solid black line.

in $\mathrm{PrMnSbO} ; T_{\mathrm{N}, \operatorname{Pr}} \approx 180 \mathrm{~K}$ in $\mathrm{PrMnAsO}_{0.95} \mathrm{~F}_{0.05}$ ) within the ab plane as $\mathrm{Mn}^{2+}$ moments reorient to within this plane $[32,33]$.

\section{B. Magnetic structure of $\mathrm{Pr}_{2} \mathrm{O}_{2} \mathrm{Fe}_{2} \mathrm{OSe}_{2}$}

Ni et al. showed that $\mathrm{Pr}_{2} \mathrm{O}_{2} \mathrm{Fe}_{2} \mathrm{OSe}_{2}$ orders AFM on cooling $\left(T_{\mathrm{N}}=88.6 \mathrm{~K}\right)$ [36], consistent with magnetic susceptibility measurements (Supplemental Material [51]). In zero-field

TABLE I. Details from Rietveld refinement using NPD data collected at $1.5 \mathrm{~K}$ for $\mathrm{Pr}_{2} \mathrm{O}_{2} \mathrm{Fe}_{2} \mathrm{OSe}_{2}$ (zero field). The refinement was carried out with the nuclear structure described by space group Immm with $a=4.0390(2) \AA, b=4.0349(1) \AA$, and $c=18.3820(8) \AA$. The magnetic scattering was fitted by a second magnetic-only phase with $a, b$, and $c$ unit cell parameters twice those of the nuclear phase and moment orientations following Fig. $1(\mathrm{e}) ; R_{\mathrm{wp}}=5.07 \%, R_{\mathrm{p}}=$ $4.83 \%$, and $\chi^{2}=10.79$.

\begin{tabular}{lcccccc}
\hline \hline Atom & Site & $x$ & $Y$ & $z$ & $U_{\text {iso }} \times 100\left(\AA^{2}\right)$ & Moment $\left(\mu_{\mathrm{B}}\right)$ \\
\hline $\mathrm{Pr}$ & $4 i$ & 0 & 0 & $0.68612(6)$ & $0.35(4)$ & \\
$\mathrm{Fe}(1)$ & $2 d$ & 0.5 & 0 & 0.5 & $0.18(1)^{\mathrm{a}}$ & $3.36(1)$ \\
$\mathrm{Fe}(2)$ & $2 b$ & 0 & 0.5 & 0.5 & $0.18(1)^{\mathrm{a}}$ & $3.36(1)$ \\
$\mathrm{Se}$ & $4 i$ & 0 & 0 & $0.09806(4)$ & $0.03(2)$ & \\
$\mathrm{O}(1)$ & $4 j$ & 0.5 & 0 & $0.7523(3)$ & $0.23(3)$ & \\
$\mathrm{O}(2)$ & $2 c$ & 0.5 & 0.5 & 0 & $0.52(4)$ & \\
\hline \hline
\end{tabular}

${ }^{\mathrm{a}} U_{\text {iso }}$ for $\mathrm{Fe}(1)$ and $\mathrm{Fe}(2)$ constrained to be the same.
NPD data, we observed a broad asymmetric peak immediately above $T_{\mathrm{N}}$ around $d=3.6 \AA$, from which the most intense magnetic Bragg reflection (211) grew (Supplemental Material [51]). The form of this peak suggests that short-range 2D magnetic order, that may be characterized by a Warren function [52], occurs immediately above the transition to three-dimensional magnetic order. No Warren peak is observed above $T_{\mathrm{N}}$ in a $5 \mathrm{~T}$ applied field (Supplemental Material [51]).

Magnetic Bragg reflections are observed on cooling below $\sim 90 \mathrm{~K}$ in zero field. These reflections, consistent with the $2 k$ magnetic structure reported for $L n_{2} \mathrm{O}_{2} \mathrm{Fe}_{2} \mathrm{OSe}_{2}(L n=$ $\mathrm{La}, \mathrm{Nd}, \mathrm{Ce})[17,37]$ and $\mathrm{Sr}_{2} \mathrm{~F}_{2} \mathrm{Fe}_{2} \mathrm{OS}_{2}$ [11], increase smoothly on cooling (Supplemental Material [51]). As for other materials, magnetic reflections are anisotropically broadened, suggesting stacking faults in the magnetic structure perpendicular to the $\mathrm{Fe}_{2} \mathrm{O}$ layers. This broadening was described by an expression for antiphase boundaries perpendicular to the

TABLE II. Selected bond distances from Rietveld refinement using NPD data collected at $1.5 \mathrm{~K}$ for $\mathrm{Pr}_{2} \mathrm{O}_{2} \mathrm{Fe}_{2} \mathrm{OSe}_{2}$ (zero field).

\begin{tabular}{ll}
\hline \hline Bond & Length $(\AA)$ \\
\hline $\mathrm{Pr}-\mathrm{O}(1)$ & $2 \times 2.313(2)$ \\
$\mathrm{Pr}-\mathrm{O}(1)$ & $2 \times 2.358(3)$ \\
$\mathrm{Fe}(1)-\mathrm{O}(2)$ & $2 \times 2.0195(1)$ \\
$\mathrm{Fe}(1)-\mathrm{Se}$ & $4 \times 2.7054(4)$ \\
$\mathrm{Fe}(2)-\mathrm{O}(2)$ & $2 \times 2.01743(8)$ \\
$\mathrm{Fe}(2)-\mathrm{Se}$ & $2 \times 2.7069(4)$ \\
\hline
\end{tabular}




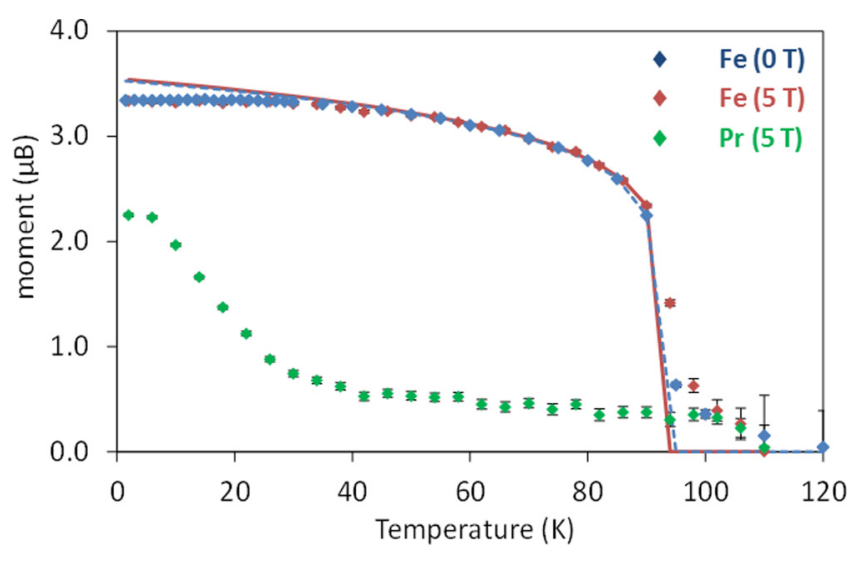

FIG. 4. Evolution of magnetic moments on Fe sites in zero applied magnetic field (blue diamonds), and in $5 \mathrm{~T}$ (red diamonds) and on Pr sites in $5 \mathrm{~T}$ (green diamonds) from sequential Rietveld refinements; dashed blue (zero field) and solid red $(5 \mathrm{~T})$ lines are guides to the eye showing critical behavior for a 2D-Ising-like system [with critical exponent $\beta \approx 0.1(1)$ ].

$c$ axis [37,53] (Supplemental Material [51]) with a magnetic correlation length along $c, \xi_{\text {c }}$, of $138(2) \AA$ at $1.5 \mathrm{~K}$. A good Rietveld fit was obtained with an Immm nuclear phase and $2 k$ magnetic ordering on the Fe sites (Fig. 3 and Tables I and II.).

Attempts to include an ordered moment on the Pr site (in zero field) in $\mathrm{Pr}_{2} \mathrm{O}_{2} \mathrm{Fe}_{2} \mathrm{OSe}_{2}$ gave no improvement in fit ( $R_{\mathrm{wp}}$ decreased by $0.0003 \%$ for this additional parameter) and a $\mathrm{Pr}^{3+}$ moment of zero within two standard uncertainties $\left[0.11(8) \mu_{\mathrm{B}}\right]$; we conclude that there is no long-range order of $\mathrm{Pr}^{3+}$ moments at $1.5 \mathrm{~K}$ in zero field. We note that the $\mathrm{Fe}^{2+}$ moment determined here is larger than that reported by $\mathrm{Ni}$ et al. [2.23(3) $\mu_{\mathrm{B}}$ at $5 \mathrm{~K}$ ] [36] (presumably due to improved fitting of broadened magnetic Bragg reflections) but is similar to that reported for other $\mathrm{Fe}_{2} \mathrm{O}$ phases $[11,17,37,53]$. Sequential Rietveld refinements carried out using the $59^{\circ}$ data bank indicate that the onset of the $2 k$ magnetic order on the
Fe sublattice (Fig. 4) is 2D-Ising-like, similar to other $\mathrm{Fe}_{2} \mathrm{O}$ phases $[17,25,37]$. The higher order terms needed to stabilize the $2 k$ structure couple the perpendicular $k$ vectors and introduce the $C_{4}$ rotational symmetry to the $L n_{2} \mathrm{O}_{2} \mathrm{Fe}_{2} \mathrm{OSe}_{2}$ magnetic symmetry [17]. These terms may compete with the $\mathrm{Pr}^{3+}$-driven orthorhombic distortion but are likely to be much smaller in energy than the Pr orbital degrees of freedom that drive the structural distortion.

$\mathrm{Ni}$ et al. suggested that $\mathrm{Pr}_{2} \mathrm{O}_{2} \mathrm{Fe}_{2} \mathrm{OSe}_{2}$ might undergo a metamagnetic phase transition at low temperatures [36], consistent with the field dependence observed in our susceptibility measurements (Supplemental Material [51]). This prompted us to use NPD to study the effect of an applied magnetic field. In a $5 \mathrm{~T}$ applied field, magnetic Bragg reflections (consistent with the $2 k$ magnetic structure) are observed at $94 \mathrm{~K}$ and increase in intensity on cooling (Supplemental Material [51]). Below $\sim 26 \mathrm{~K}$ in a $5 \mathrm{~T}$ field, additional magnetic scattering is observed at $k=(000)$ positions (relative to the nuclear cell). These reflections are sharper than those due to the $2 k$ ordering (Fig. 5). Attempts to fit the low temperature 5 T NPD data with various ordering patterns on the Fe sublattice were unsuccessful but including a FM component on $\operatorname{Pr}^{3+}$ sites did improve the fit. 1.5 K NPD data collected in a $5 \mathrm{~T}$ field were fitted with $2 k$ magnetic ordering on the $\mathrm{Fe}$ sublattice (with anisotropic peak broadening of these reflections as described above) and with $\mathrm{Pr}^{3+}$ moments along the shorter $b$ axis (Supplemental Material [51]). The same peak shape was used to fit nuclear reflections and the additional reflections arising from the FM component, suggesting no stacking faults for this fieldinduced FM component. The $\operatorname{Pr}^{3+}$ moment at $5 \mathrm{~T}$ [2.21(1) $\left.\mu_{\mathrm{B}}\right]$ is smaller than that found in PrMnSbO [2.96(3) $\left.\mu_{\mathrm{B}}\right]$ and $\mathrm{PrMnAsO}_{0.95} \mathrm{~F}_{0.05}\left[\sim 3 \mu_{\mathrm{B}}\right]$ [33] but larger than that found in the poorly metallic PrFeAsO [0.83(9) $\mu_{\mathrm{B}}$ ] [54].

Whilst this model gives a good fit to our NPD data, it may not correspond to the ground state magnetic structure in an applied magnetic field due to the difficulties associated with working with powder samples and significant spin anisotropy.

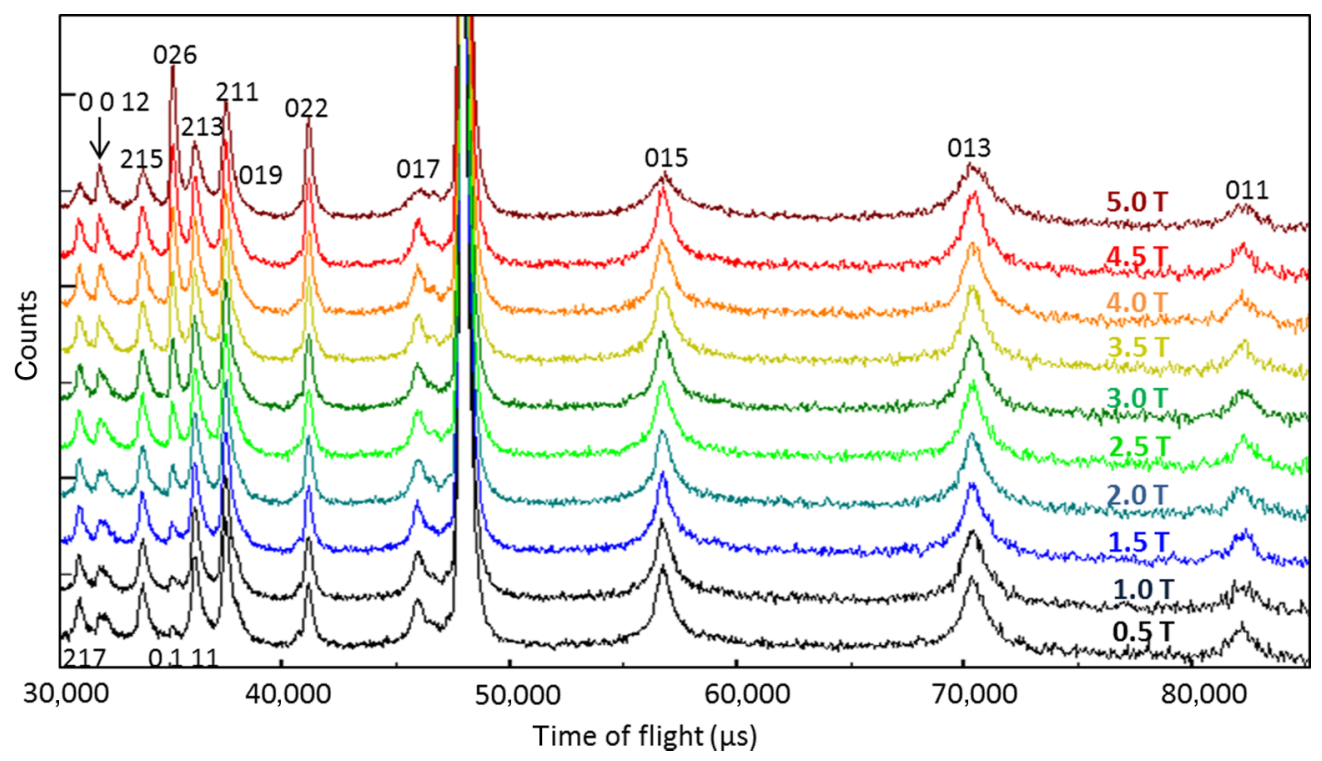

FIG. 5. Fifty-nine-degree bank NPD data collected for $\mathrm{Pr}_{2} \mathrm{O}_{2} \mathrm{Fe}_{2} \mathrm{OSe} e_{2}$ at $2 \mathrm{~K}$ in an applied magnetic field showing evolution of magnetic Bragg reflections $(022,026)$ as a function of applied field, as well as broadening of existing $2 k$ magnetic reflections (e.g., 015, 017). 

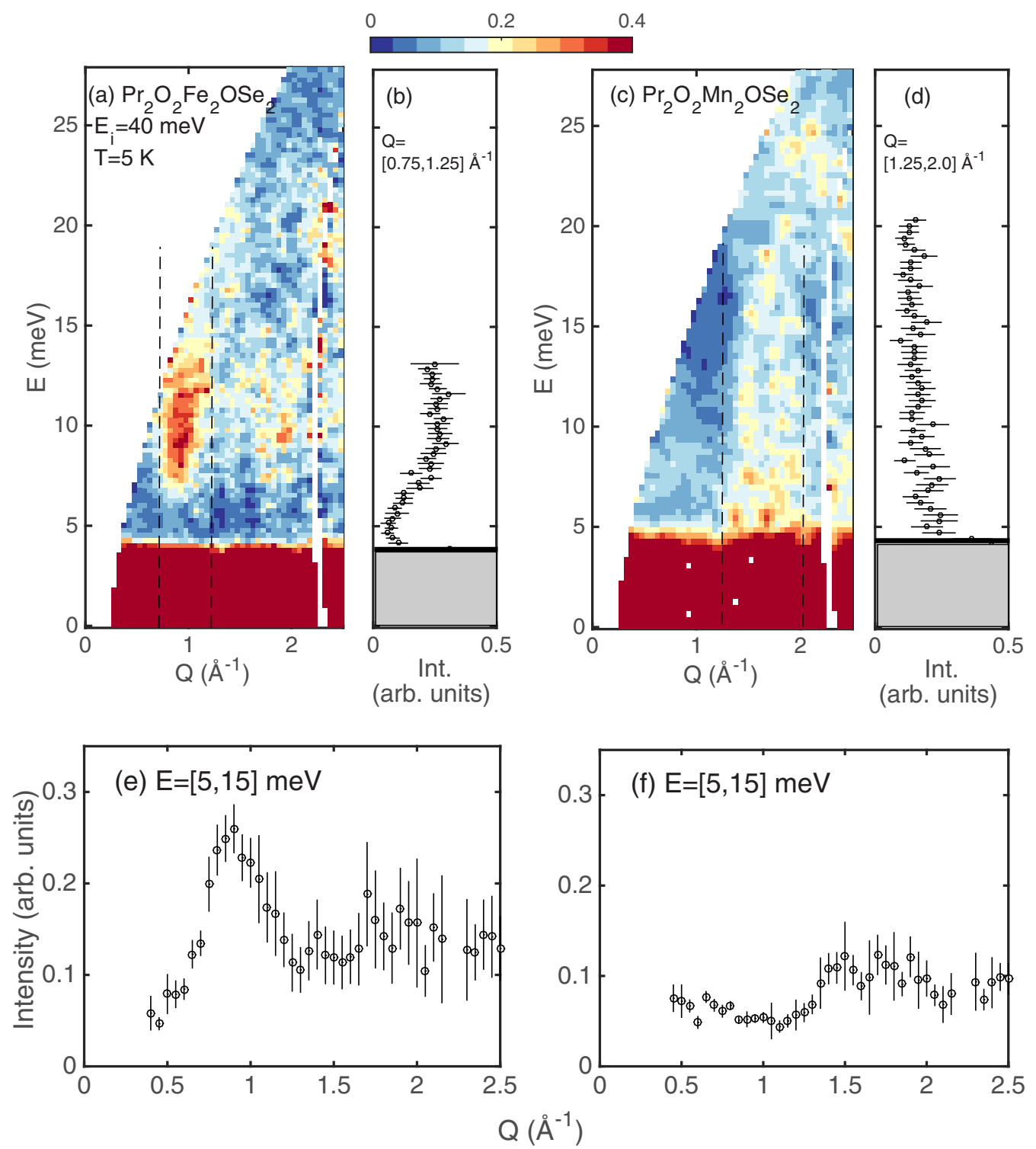

FIG. 6. (a) Energy momentum slice for $\operatorname{Pr}_{2} \mathrm{O}_{2} \mathrm{Fe}_{2} \mathrm{OSe}_{2}$ at $5 \mathrm{~K}$, (b) a constant momentum cut integrating over $Q=[0.75,1.25] \AA^{-1}$, (c) energy momentum slice for $\operatorname{Pr}_{2} \mathrm{O}_{2} \mathrm{Mn}_{2} \mathrm{OSe}_{2}$ at $5 \mathrm{~K}$, (d) a constant momentum cut integrating over $Q=[1.25,2.00] \AA^{-1}$; constant energy slices are shown for $\mathrm{Pr}_{2} \mathrm{O}_{2} \mathrm{Fe}_{2} \mathrm{OSe}_{2}$ (e) and for $\mathrm{Pr}_{2} \mathrm{O}_{2} \mathrm{Mn}_{2} \mathrm{OSe}_{2}$ (f) integrating over [5,15] meV. The area contaminated by incoherent scattering from the $E=0$ elastic line is shaded in grey in panels (b) and (d).

Although crystallites in our pelletized sample are unlikely to reorient in the applied (uniaxial) field, the powder averaging inherent in a "powder diffraction" experiment is likely to break down: the applied magnetic field will cause moments to reorient depending on the relative direction of the applied field, orientation of the crystallite, and direction of any spin anisotropy (particularly relevant to the $\mathrm{Pr}^{3+}$ sites). A single crystal sample would be needed to overcome these effects.

\section{Transition metal magnetic excitations in $\operatorname{Pr}_{2} \mathrm{O}_{2} \mathrm{Fe}_{2} \mathrm{OSe}_{2}$ and $\mathrm{Pr}_{2} \mathrm{O}_{2} \mathrm{Mn}_{2} \mathrm{OSe}_{2}$}

In this section we discuss the excitations on the transition metal site in $\mathrm{Pr}_{2} \mathrm{O}_{2} \mathrm{Fe}_{2} \mathrm{OSe}_{2}$ and the manganese analogue $\mathrm{Pr}_{2} \mathrm{O}_{2} \mathrm{Mn}_{2} \mathrm{OSe}_{2}$ (which shows similar but more marked orthorhombicity at low temperatures). In the following section we will present the localized excitations from the crystalline electric field on the $\mathrm{Pr}^{3+}$ site. The magnetic excitations for $\mathrm{Pr}_{2} \mathrm{O}_{2} \mathrm{Fe}_{2} \mathrm{OSe}_{2}$ and $\mathrm{Pr}_{2} \mathrm{O}_{2} \mathrm{Mn}_{2} \mathrm{OSe}_{2}$ are shown in Fig. 6 measured on the MARI spectrometer at $5 \mathrm{~K}$ with $E_{i}=40 \mathrm{meV}$ (the same configuration used to study $\mathrm{La}_{2} \mathrm{O}_{2} \mathrm{Fe}_{2} \mathrm{OSe}_{2}$ [17]). The large momentum dependence of the intensity [Figs. 6(a) and 6(c)] indicates that it is due to magnetic excitations associated with the $\mathrm{Fe}^{2+}$ or $\mathrm{Mn}^{2+}$ sites rather than the $\mathrm{Pr}^{3+}$ site. As discussed for $\mathrm{Ce}_{2} \mathrm{O}_{2} \mathrm{FeSe}_{2}$ [24], low-energy transition metal and CEFs can be distinguished by their momentum dependence, with excitations associated with the lanthanide site $\left(\mathrm{Pr}^{3+}\right.$ in this case), local CEFs showing no strong momentum dependence.

We consider first $\mathrm{Pr}_{2} \mathrm{O}_{2} \mathrm{Fe}_{2} \mathrm{OSe}_{2}$ [Figs. 6(a), 6(b), and 6(e)]. The magnetic cross section [Fig. 6(a)] is almost identical to that observed for $\mathrm{La}_{2} \mathrm{O}_{2} \mathrm{Fe}_{2} \mathrm{OSe}_{2}$ [17] (further supporting our 
assignment of the fluctuations to the $\mathrm{Fe}^{2+}$ site rather than the $\mathrm{Pr}^{3+}$ site). The low-energy excitation spectrum is dominated by an anisotropy gap. Due to powder averaging and the 2D nature of the magnetic fluctuations, the gapped excitations manifest as a broadened "step" in the excitation spectrum rather than a sharp peak. This has been observed for other systems, including the sum rule analysis of neutron scattering data for $\mathrm{La}_{2} \mathrm{O}_{2} \mathrm{Fe}_{2} \mathrm{OSe}_{2}$ [17] (see Fig. 3(d) of Ref. [17]), as well as for low-dimensional magnets $\alpha-\mathrm{NaMnO}_{2}$ (see Fig. 2 of Ref. [55]) and $\mathrm{Cu}$ (quinoxaline) $\mathrm{Br}_{2}$ (see Fig. 7 of Ref. [56]). The similar magnetic cross sections for $\mathrm{Pr}_{2} \mathrm{O}_{2} \mathrm{Fe}_{2} \mathrm{OSe}_{2}$ and $\mathrm{La}_{2} \mathrm{O}_{2} \mathrm{Fe}_{2} \mathrm{OSe}_{2}$ also suggest that $\mathrm{Pr}_{2} \mathrm{O}_{2} \mathrm{Fe}_{2} \mathrm{OSe}_{2}$ has exchange interactions similar to $\mathrm{La}_{2} \mathrm{O}_{2} \mathrm{Fe}_{2} \mathrm{OSe}_{2}$, which were modeled in terms of dominant AFM Fe-O-Fe $J_{2^{\prime}}$ interactions and weaker FM FeSe-Fe $J_{2}$ interactions (spectra compared against calculations with $J_{1}=0.75 \mathrm{meV}, J_{2}=-0.10 \mathrm{meV}$ and $J_{2^{\prime}}=1.00 \mathrm{meV}$ ). A large anisotropy results in a gap in the momentum slice [Fig. 6(a)], and this is confirmed in the momentum integrating cut [Fig. 6(b)], which shows an onset of magnetic intensity at $\sim 5-6 \mathrm{meV}$. The similar magnetic behavior of the iron sublattice in both La and $\mathrm{Pr}$ analogues highlights the limited influence of the lanthanide ion (size and magnetic properties) on the robust $2 k$ magnetic order on the iron sublattice [37].

The magnetic response in $\mathrm{Pr}_{2} \mathrm{O}_{2} \mathrm{Mn}_{2} \mathrm{OSe}_{2}$ [Figs. 6(c), 6(d), and 6(f)] differs from the $\mathrm{Fe}^{2+}$ analogue, displaying a more complex momentum dependence consistent with several strong and competing exchange interactions within the $\mathrm{Mn}$ sublattice $[14,15]$. This is consistent with the frustrated nature of $\mathrm{Mn}_{2} \mathrm{O}$ materials suggested by NPD analysis and property measurements $[13,15]$. The bulk of the spectral weight in the $\mathrm{Mn}^{2+}$ analogue is shifted to larger momentum transfers [Fig. 6(f)], indicating an exchange interaction over a smaller length scale becoming dominant [57]. There is also significant spectral weight in the limit $\mathrm{Q} \rightarrow 0$, which may indicate multiple competing exchange interactions corresponding to different length scales. On the scale of the resolution (full width at half maximum $\approx 2.2 \mathrm{meV}$ ), no anisotropy gap is observed for $\mathrm{Pr}_{2} \mathrm{O}_{2} \mathrm{Mn}_{2} \mathrm{OSe}_{2}$, and the momentum integrating cut [Fig. 6(d)] is smooth and almost independent of energy, in contrast with that for $\operatorname{Pr}_{2} \mathrm{O}_{2} \mathrm{Fe}_{2} \mathrm{OSe}_{2}$ [Fig. 6(b)]. This is consistent with the more three-dimensional character of the onset of $\mathrm{Mn}^{2+}$ magnetic ordering suggested by NPD analysis [critical exponent $\beta=0.24$ (3) for $\mathrm{La}_{2} \mathrm{O}_{2} \mathrm{Mn}_{2} \mathrm{OSe}_{2}$, compared with 0.122(1) for $\left.\mathrm{La}_{2} \mathrm{O}_{2} \mathrm{Fe}_{2} \mathrm{OSe}_{2}\right][14,17]$.

\section{Linear spin wave analysis to determine exchange interactions in $\mathrm{Pr}_{2} \mathrm{O}_{2} \mathrm{Mn}_{2} \mathrm{OSe}_{2}$}

To compare these $\mathrm{Fe}^{2+}$ and $\mathrm{Mn}^{2+}$ oxyselenides, linear spin wave calculations were carried out with the aim of reproducing the features which distinguish the $\mathrm{Mn}^{2+}$ phase from the $\mathrm{Fe}^{2+}$ analogue. The calculations were done using the SpinW package, based on the Heisenberg Hamiltonian

$$
\mathcal{H}=\sum_{i j} J_{i j} \overrightarrow{S_{i}} \cdot \overrightarrow{S_{j}}
$$

where $i, j$ are magnetic sites and $J_{i j}$ is the coupling between them $(J<0$ indicates FM exchange whilst $J>0$ indicates AFM exchange). These calculations, to investigate the magnetic excitations from the $\mathrm{Mn}^{2+}$ sublattice, were performed on

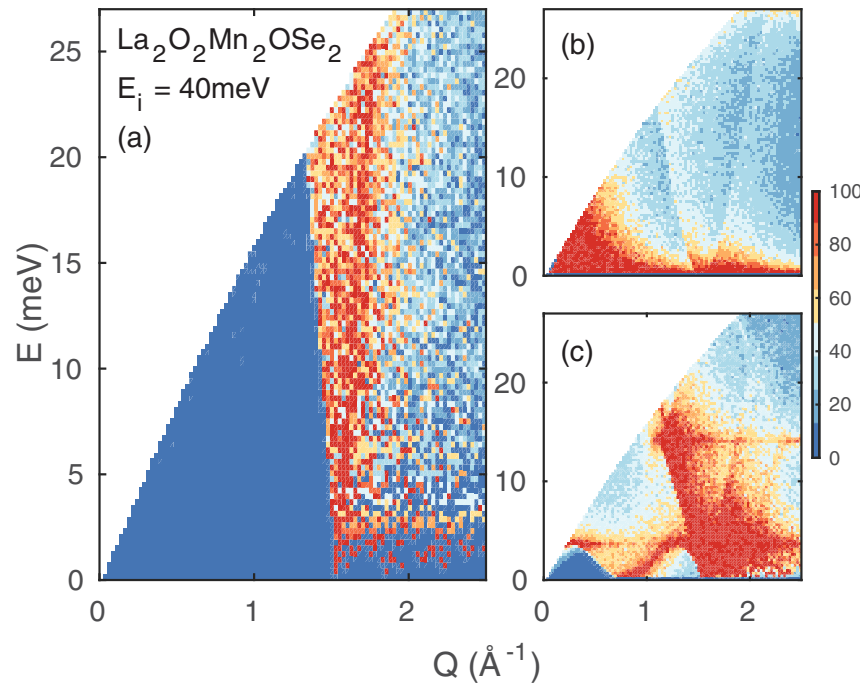

FIG. 7. Simulated INS powder spectra for $\mathrm{La}_{2} \mathrm{O}_{2} \mathrm{Mn}_{2} \mathrm{OSe}_{2}$ using SpinW with exchange constants (a) $J_{1}=5.05 \mathrm{meV}$ and $J_{2}=J_{2^{\prime}}=0$; (b) $J_{2}=-2.8 \mathrm{meV}$ and $J_{1}=J_{2^{\prime}}=0$, and (c) $J_{1}=J_{2^{\prime}}=1.4 \mathrm{meV}$ and $J_{2}=-1.4 \mathrm{meV}$.

$\mathrm{La}_{2} \mathrm{O}_{2} \mathrm{Mn}_{2} \mathrm{OSe}_{2}$ (excitations from the $\mathrm{Pr}^{3+}$ sublattice are not relevant for this analysis, as explained above).

Given the complex neutron response and the number of exchange interactions, general fits to the inelastic spectra were not possible; we therefore investigated the effect of each exchange parameter on the neutron response (Fig. 7). A dominant $J_{1}$ interaction reproduced the "wall" of scattering at larger momentum transfers [Fig. 7(a)], consistent with the experimentally observed magnetic structure $[14,15]$. The tuning of the nnn exchanges $J_{2}$ and $J_{2^{\prime}}$ gives a shift of the magnetic spectral weight, which is inconsistent with the observed spectra [Fig. 6(c)]. From this, we conclude that the strong AFM nn exchange $J_{1}$ is dominant.

The effect of tuning nnn interactions $J_{2}(\mathrm{Mn}-\mathrm{Se}-\mathrm{Mn})$ and $J_{2^{\prime}}\left(\mathrm{Mn}-\mathrm{O}-\mathrm{Mn}\right.$ ) with fixed AFM $J_{1}$ are investigated in Fig. 8. There is some ambiguity from theoretical papers as to the sign of the nnn Mn-Se-Mn $J_{2}$ interaction [13,58], but our model considers FM $J_{2}$ exchange, reflecting the experimental magnetic structure. Figures 8(a)-8(c) illustrate the effect of increasing the FM $J_{2}$ on the neutron response: little qualitative change is observed, and we cannot determine the value of FM $J_{2}$ from this analysis. Figures $8($ d)-8(f) illustrate the effect of introducing AFM $J_{2^{\prime}}\left(180^{\circ} \mathrm{Mn}-\mathrm{O}-\mathrm{Mn}\right.$ exchange) on the neutron response, and we note that it is in competition with AFM nn $J_{1}$ : both these AFM interactions cannot be satisfied simultaneously. On introducing $J_{2^{\prime}}$ [Figs. 8(d)-8(f)], we see a shift in spectral weight to lower energies and to lower momentum transfers. While this may be consistent with the presence of spectral weight in the limit $Q \rightarrow 0$ [Fig. 6(f)], $J_{2^{\prime}}$ does seem to reduce the "wall" of scattering at larger momentum transfers. Based on this comparison of our experimental data with linear spin wave calculations, we estimate values for the exchange interactions $\left(J_{1} \approx 5 \mathrm{meV}, J_{2} \approx-5 \mathrm{meV}\right.$, and $J_{2^{\prime}} \approx 1-5 \mathrm{meV}$ ), which are consistent with the higher degree of magnetic frustration for the Mn (compared with, 


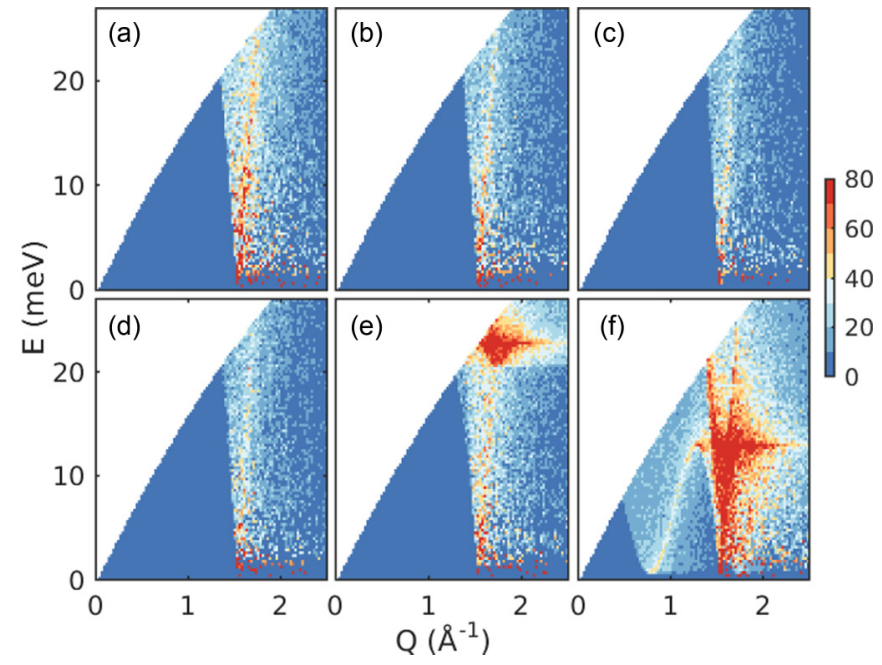

FIG. 8. SpinW simulated INS spectra simulated for $\mathrm{La}_{2} \mathrm{O}_{2} \mathrm{Mn}_{2} \mathrm{OSe}_{2}$ with fixed $\mathrm{nn} J_{1}=5.05 \mathrm{meV}$. Top row has fixed $J_{2^{\prime}}=0$ and $J_{2}=-1,-3$, and $-5 \mathrm{meV}$ for (a), (b), and (c), respectively. Bottom row has fixed $J_{2}=-5 \mathrm{meV}$ and $J_{2^{\prime}}=1,3$, and $5 \mathrm{meV}$ for (d), (e), and (f), respectively.

e.g., $M=\mathrm{Fe}$ ) systems expected from analysis of diffraction data and heat capacity measurements $[13,15]$.

\section{D. $\operatorname{Pr}^{3+}$ CEFs}

Having discussed excitations associated with the transition metal sublattice, we now discuss the local electronic crystal field probed through excitations on the $\operatorname{Pr}^{3+}$ site. Given the local symmetry of the $\mathrm{Pr}^{3+}$ site [the $4 e$ site of $C_{4 v}(4 \mathrm{~mm})$ symmetry] in the tetragonal $I 4 / \mathrm{mmm}$ phase, the Hamiltonian associated with the crystalline electric field takes the form described by Stevens operators [59]:

$$
H_{\text {tet }}=B_{2}^{0} O_{2}^{0}+B_{2}^{2} O_{2}^{2}+B_{4}^{0} O_{4}^{0}+B_{4}^{4} O_{4}^{4}+B_{6}^{0} O_{6}^{0}+B_{6}^{4} O_{6}^{4}
$$

The orthorhombic distortion lowers the symmetry of the $\mathrm{Pr}^{3+}$ site [to $C_{2 v}(\mathrm{~mm} 2)$ symmetry at the $4 i$ site of the orthorhombic Immm model], and additional terms are needed in the Hamiltonian to describe the crystal field:

$$
\begin{aligned}
H_{\text {ortho }}= & B_{2}^{0} O_{2}^{0}+B_{2}^{2} O_{2}^{2}+B_{4}^{0} O_{4}^{0}+B_{4}^{2} O_{4}^{2}+B_{4}^{4} O_{4}^{4} \\
& +B_{6}^{0} O_{6}^{0}+B_{6}^{2} O_{6}^{2}+B_{6}^{4} O_{6}^{4}+B_{6}^{6} O_{6}^{6}
\end{aligned}
$$

We now discuss how these terms affect the neutron scattering cross section. In the dipole approximation for localized magnetic moments, the neutron scattering cross section at small momentum transfers can be written [60]

$$
\begin{aligned}
\frac{d^{2} \sigma}{d \Omega d \omega}= & \frac{\left(\gamma r_{0}\right)^{2}}{4} \frac{k_{f}}{k_{i}} f^{2}(Q) \\
& \times \sum_{r, s} \rho_{n}\left|\left\langle r\left|J_{\perp}\right| s\right\rangle\right|^{2} \delta\left(E_{r}-E_{s}-\hbar \omega\right)
\end{aligned}
$$

where $\frac{\left(\gamma r_{0}\right)^{2}}{4}$ is 73 mbarns sr ${ }^{-1}, f^{2}(Q)$ is the magnetic form factor, the indices $r, s$ refer to the different crystalline field wave functions, $\rho_{n}$ is a matrix element weighting factor, and $J_{\perp}$ is the projection of $J$ perpendicular to $Q$. This cross section
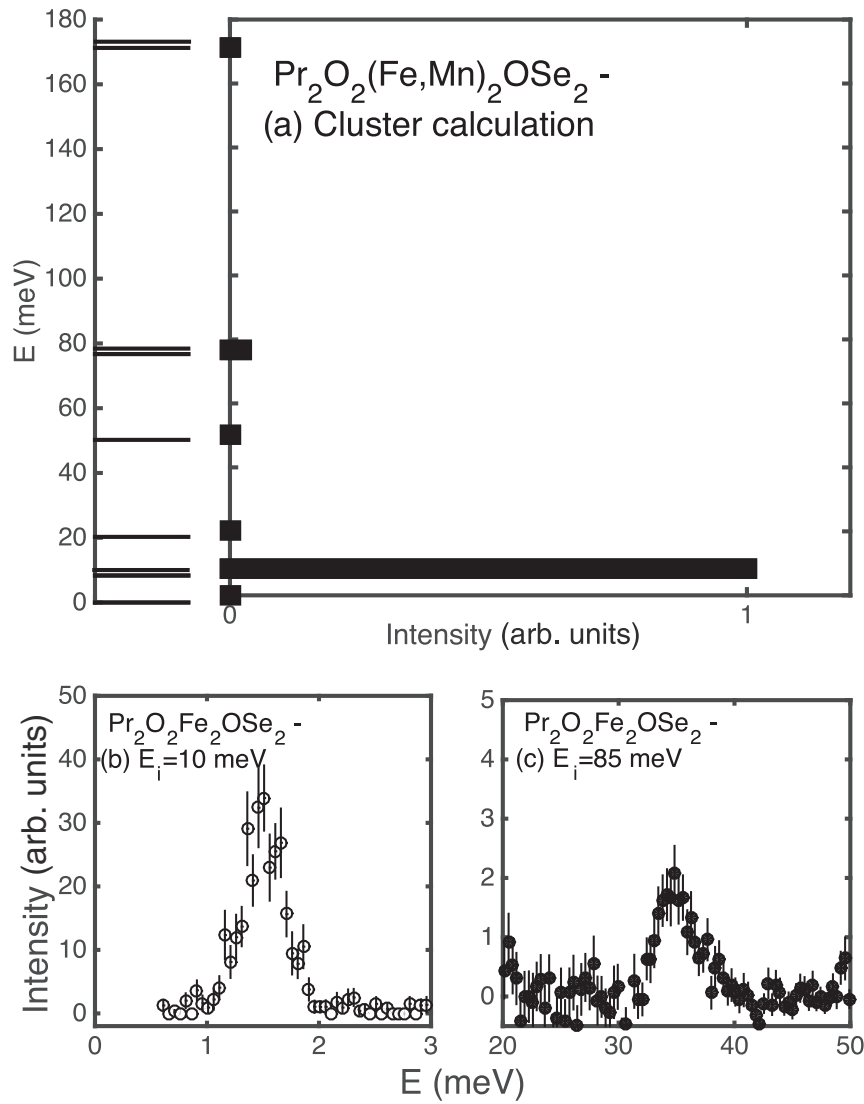

FIG. 9. (a) Calculated crystal field scheme for $\operatorname{Pr}^{3+}$ ions in $\mathrm{Pr}_{2} \mathrm{O}_{2} M_{2} \mathrm{OSe}_{2}(M=\mathrm{Mn}, \mathrm{Fe})$ assuming a point charge model summing over 10 tetragonal unit cells. The calculation shows three allowed transitions, two of which are observed and are shown in (b) and (c), which show energy scans integrating in momentum over $Q=[0,5] \AA^{-1}$ ) for $\operatorname{Pr}_{2} \mathrm{O}_{2} \mathrm{Fe}_{2} \mathrm{OSe}_{2}$. The third, high-energy crystal field level was not observed experimentally, consistent with the weak cross section expected from the point charge calculation.

illustrates a selection rule $\Delta m=0, \pm 1$ (where $m$ are the eigenvalues of the $J_{\perp}$ operator). (Magnetic CEFs are distinguished from collective excitations associated with exchange interactions between magnetic $\mathrm{Fe}^{2+}$ ions by their momentum dependence, as discussed above and for $\mathrm{Ce}_{2} \mathrm{O}_{2} \mathrm{FeSe}_{2}$ [24]).

We have performed a point charge calculation for the crystal field Hamiltonian with $\operatorname{Pr}^{3+}(J=4)$ outlined in Eq. (2). The results of this "cluster" point charge calculation are shown in Fig. 9, with energy positions and calculated neutron scattering dipole intensities displayed. The calculation was performed considering localized point charges with Stevens parameters obtained by summing over 10 unit cells to ensure convergence [61]. This approximate approach is justified by the fact that Stevens parameters depend on sums that decay rapidly with radius $R$ as $\sim 1 / R^{(L+1)(2 L+1)}$ with $L=2,4,6$, facilitating a rapid convergence. These calculations give similar results to those on tetragonal $\mathrm{Pr}_{2} \mathrm{CuO}_{4}$ with a singlet ground state and a first excited state with a large calculated and measured neutron cross section [62-64]. We note that while the crystal field levels are either singly or doubly degenerate, the wave function is a linear combination of the nine eigenstates of $J_{z}$ associated with a total angular momentum $J=4$. While the point charge 
model is not entirely appropriate for these oxyselenides (in which covalency is likely to be important), it does provide a useful starting point for understanding the properties of the CEFs and the degeneracy.

The results show that the lowest energy CEF from the singlet ground state is a doublet, and this excitation is calculated to have the largest neutron scattering intensity. Two other excitations were calculated to have finite neutron cross sections and are also illustrated in Fig. 9, though with considerably weaker intensities. $\mathrm{Pr}^{3+}$ is a non-Kramers system with $J=4$; therefore, the splitting of the excitations is sensitive to the crystal field local symmetry. In particular, on including terms in the Hamiltonian listed in Eq. (3) for an orthorhombic unit cell, splitting of the doublet CEFs is recovered. This is in contrast to the Kramers system involving $\mathrm{Ce}^{3+}$, where Kramers theorem guarantees that the excitation spectrum consists of doublets that cannot be split unless a magnetic field is applied, as is the case for a magnetically ordered system, as demonstrated by neutron scattering studies for CeFeAsO [41]. On inclusion of terms allowed for orthorhombic symmetry [terms included in $H_{\text {ortho }}$, Eq. (3)], this doublet splits, making this lowest energy excitation sensitive to local electrical inhomogeneity of local distortions.

The lowest two CEFs for $\mathrm{Pr}^{3+}$ in $\mathrm{Pr}_{2} \mathrm{O}_{2} \mathrm{Fe}_{2} \mathrm{OSe}_{2}$ are shown in Figs. 9(b) and 9(c). Only two CEFs are observed in this energy range, consistent with the point charge "cluster" calculations described above. The most intense excitation occurs at low energies $(\sim 1.5 \mathrm{meV})$, and the next highest excitation occurs at $\sim 35 \mathrm{meV}$. These two excitations have intensities similar to those predicted from the point charge cluster calculations but appear at significantly lower energies than predicted by the calculation. This difference in observed and calculated energies is presumably due to orbital overlap and covalency, which requires a more detailed electronic theory than that afforded by the point charge model. The crystal field energies, level scheme, and neutron cross sections measured here for $\mathrm{Pr}_{2} \mathrm{O}_{2} \mathrm{Fe}_{2} \mathrm{OSe}_{2}$ are consistent with those for $\mathrm{Pr}^{3+}$ in cuprate systems [28-30], including $\mathrm{Pr}_{2} \mathrm{CuO}_{4}$.

Given the strong neutron scattering intensity of the lowest energy $\mathrm{Pr}^{3+}$ excited doublet, and its sensitivity to local ligand fields, we studied this excitation as a function of temperature using the MARI spectrometer with $E_{i}=10 \mathrm{meV}$ (Fig. 10). Figure 10(a) illustrates a resolution-limited excitation at low temperatures $(3.5 \mathrm{~K})$ which broadens on warming [Figs. 10(b) and $10(\mathrm{c})$ at $40 \mathrm{~K}$ and $75 \mathrm{~K}$, respectively]. The linewidth and energy position are shown in Figs. 10(d) and 10(e). Both parameters show a change at $30-50 \mathrm{~K}$ and then a saturation below $\sim 20 \mathrm{~K}$. At the lowest temperatures, the first CEF of $\mathrm{Pr}_{2} \mathrm{O}_{2} \mathrm{Fe}_{2} \mathrm{OSe}_{2}$ is resolution limited, and no splitting (from the subtle crystallographic orthorhombic distortion) is observed.

The effects of an applied magnetic field on $\operatorname{Pr}_{2} \mathrm{O}_{2} \mathrm{Fe}_{2} \mathrm{OSe}_{2}$ were investigated using this point charge "cluster" model described above by adding a Zeeman term to the Hamiltonian given in Eq. (3). A weak transverse magnetic field splits the $\mathrm{Pr}^{3+}$ excited doublet and results in a weak $\mathrm{Pr}^{3+}$ transverse moment (typically $\sim 1 \mu_{\mathrm{B}}$ per $\mathrm{Pr}^{3+}$ site for a $5 \mathrm{~T}$ field). Calculations with the external field perpendicular to the (001) planes did not give any such mixing and therefore resulted in a zero $\mathrm{Pr}^{3+}$ moment. The experimental observation of an ordered in-plane $\mathrm{Pr}^{3+}$ moment in an applied magnetic field for
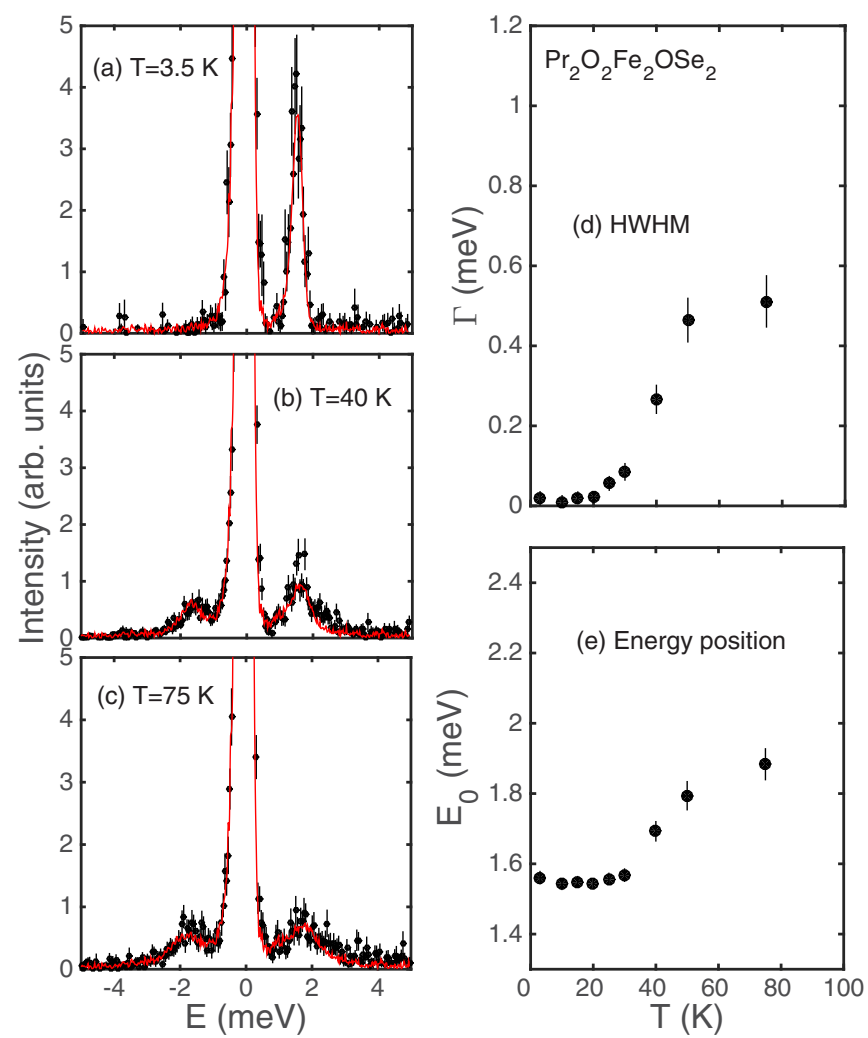

FIG. 10. The lowest energy crystal field excitation in $\mathrm{Pr}_{2} \mathrm{O}_{2} \mathrm{Fe}_{2} \mathrm{OSe}_{2}$ : the linewidth and energy of the excitation are shown as a function of temperature [panels (d) and (e), respectively]. $\mathrm{HWHM}=$ half width at half maximum.

$\mathrm{Pr}_{2} \mathrm{O}_{2} \mathrm{Fe}_{2} \mathrm{OSe}_{2}$ (as described above in a $5 \mathrm{~T}$ field, see Figs. 4 and 5 and Supplemental Material [51]) is consistent with our calculations and CEF understanding.

The temperature dependences of the low-energy $\mathrm{Pr}^{3+}$ crystal fields for $\mathrm{Pr}_{2} \mathrm{O}_{2} \mathrm{Mn}_{2} \mathrm{OSe}_{2}$ are shown in Fig. 11. In contrast to the $\mathrm{Fe}^{2+}$ analogue discussed above, at $3.5 \mathrm{~K}$, the low-energy excitation [Fig. 11(a)] shows two distinct components, with a sharp component at $\sim 2.8 \mathrm{meV}$ and an overdamped component at $E=0$. The excitation was fitted using two Lorentzian peaks at low temperatures. At high temperatures, only one temporally overdamped mode is observed, and so only one overdamped Lorentzian term was used to fit these higher temperature data. The linewidth as a function of temperature is shown in Fig. 11(d) for the temperature range for which two Lorentzian terms were used. The overdamped component is observed even at the lowest temperatures [Fig. 11(d)], in contrast to the $\mathrm{Fe}^{2+}$ analogue for which the temporally sharp (resolution-limited in energy) CEFs are observed at low temperatures. From our data for $\mathrm{Pr}_{2} \mathrm{O}_{2} \mathrm{Mn}_{2} \mathrm{OSe}_{2}$, it is not possible to determine whether the overdamped component is due to splitting of the first excited doublet or due to a broadening in energy of the lowest energy ground state. The broad excitation observed for $\mathrm{Pr}_{2} \mathrm{O}_{2} \mathrm{Mn}_{2} \mathrm{OSe}_{2}$ may allow a nonzero static moment on $\mathrm{Pr}^{3+}$ sites through mixing of the lowest energy crystal fields. It is interesting that there is no evidence for long-range $\operatorname{Pr}^{3+}$ magnetic order in $\operatorname{Pr}_{2} \mathrm{O}_{2} M_{2} \mathrm{OSe}_{2}$ whilst $\mathrm{Pr}^{3+}$ moments do order in several related 1111 materials, including $\mathrm{PrFeAsO}$ [31], PrMnAsO [33], and PrMnSbO [32], in which the $\operatorname{Pr}^{3+}$ 

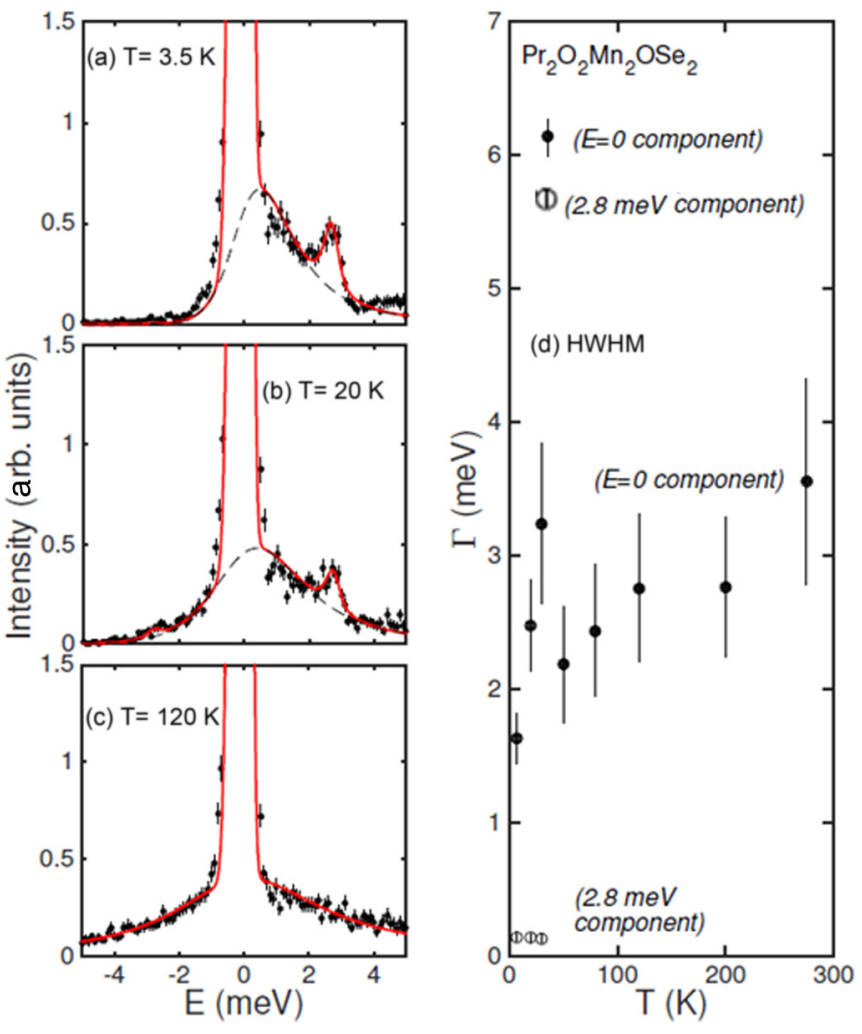

FIG. 11. The lowest energy CEF in $\mathrm{Pr}_{2} \mathrm{O}_{2} \mathrm{Mn}_{2} \mathrm{OSe}_{2}$ : the linewidth of the excitation is shown as a function of temperature [panel (d)].

order is often coupled to the transition metal magnetism. A static $\mathrm{Pr}^{3+}$ moment $\left(\sim 3 \mu_{\mathrm{B}}\right.$ at $\left.2 \mathrm{~K}\right)$ was observed in PrMnAsO ${ }_{0.95} \mathrm{~F}_{0.05}$ [33], which has a smaller orthorhombic distortion; we cannot rule out the possibility of a small localized moment on $\operatorname{Pr}^{3+}$ sites in $\operatorname{Pr}_{2} \mathrm{O}_{2} \mathrm{Mn}_{2} \mathrm{OSe}_{2}$ that our NPD data are not sensitive to, and further studies, for example by muon spin relaxation $(\mu \mathrm{SR})$, would be of interest.

\section{DISCUSSION}

From INS experiments, we have observed significant temporal broadening of the lowest energy $\mathrm{Pr}^{3+}$ ligand field excitations at all temperatures in $\mathrm{Pr}_{2} \mathrm{O}_{2} \mathrm{Mn}_{2} \mathrm{OSe}_{2}$, and only at temperatures above $\sim 30-50 \mathrm{~K}$ in $\mathrm{Pr}_{2} \mathrm{O}_{2} \mathrm{Fe}_{2} \mathrm{OSe}_{2}$. Strong dampening of CEFs has been observed previously in several systems, including $\mathrm{PrOs}_{4} \mathrm{Sb}_{12}$ [65] and Gd-based metals [66], and theories for this broadening and its temperature dependence have been developed in the context of $\mathrm{PrTl}_{3}$ [67]. However, all these theories involve a coupling to the electronic degrees of freedom and are difficult to apply to the $\mathrm{Pr}_{2} \mathrm{O}_{2} \mathrm{M}_{2} \mathrm{OSe}_{2}$ oxyselenides, which are semimetallic/insulating with energy gaps of $\sim 1 \mathrm{eV}$. Any coupling between crystal electric field excitations and electronic degrees of freedom in $\mathrm{Pr}_{2} \mathrm{O}_{2} \mathrm{M}_{2} \mathrm{OSe}_{2}$ materials are unlikely to be on the millielectronvolt energy scale probed in our experiments.

As discussed above, NPD experiments indicate that $\mathrm{Pr}_{2} \mathrm{O}_{2} M_{2} \mathrm{OSe}_{2}(M=\mathrm{Mn}, \mathrm{Fe})$ undergo distortions from tetragonal to orthorhombic symmetry at low temperatures driven by the non-Kramers $\mathrm{Pr}^{3+}$ ion, with the distortion of the $\mathrm{Fe}^{2+}$ analogue much smaller than for the $\mathrm{Mn}^{2+}$ phase. Such an orthorhombic distortion would split the lowest energy excited crystal field doublet, which may manifest as a broadening of the lowest energy CEF. However, no such broadening or splitting is observed in the low-temperature data for $\mathrm{Pr}_{2} \mathrm{O}_{2} \mathrm{Fe}_{2} \mathrm{OSe}_{2}$, and broadening is only observed in its higher temperature tetragonal phase. Significant broadening is observed for $\operatorname{Pr}_{2} \mathrm{O}_{2} \mathrm{Mn}_{2} \mathrm{OSe}_{2}$ at all temperatures with an overdamped component at the lowest temperatures studied. It is therefore difficult to explain the experimental measurements of the crystal fields for these materials in terms of their structural behavior.

High-temperature splitting of degenerate CEFs has been observed for $\mathrm{CeAl}_{2}[68]$ and was attributed to a dynamic second order Jahn-Teller effect. Whilst it may be tempting to attribute the high-temperature broadening for $\operatorname{Pr}_{2} \mathrm{O}_{2} \mathrm{Fe}_{2} \mathrm{OSe}_{2}$ to such effects, it is not consistent with the $\mathrm{Pr}_{2} \mathrm{O}_{2} \mathrm{Mn}_{2} \mathrm{OSe}_{2}$ data, for which broadening was observed at all temperatures. Similar high-temperature splitting has also been observed for $\mathrm{PrFeAsO}$ [46], and fluorine-doped $\mathrm{PrFeAsO}_{1-x} \mathrm{~F}_{x}$ showed broadened and split CEFs due to electrical inhomogeneity or possible local electronic disorder [69]. However, this explanation is also inconsistent with the $\mathrm{Pr}_{2} \mathrm{O}_{2} M_{2} \mathrm{OSe}_{2}$ properties discussed in this paper, which have a high degree of structural order and are stoichiometric or very close to stoichiometric (from high resolution NPD experiments described here and in Ref. [14]).

Another possible mechanism for the observed broadening is coupling between ligand field excitations and magnon excitations on the transition metal sites, as observed for $\mathrm{Tb}_{3} \mathrm{Fe}_{5} \mathrm{O}_{12}$ (Ref. [70] and Supplemental Material [51]). Magnon excitations could provide decay channels analogous to electronic excitations [67], given constraints due to momentum and energy transfer. These conditions are met in $\mathrm{Pr}_{2} \mathrm{O}_{2} \mathrm{Mn}_{2} \mathrm{OSe}_{2}$ at all temperatures, as $\mathrm{Mn}^{2+}$ magnon excitations extend to low-energy transfers (limited by the resolution) and result in spectral weight as $Q \rightarrow 0$ due to the competing exchange interactions. These magnon excitations could therefore provide decay routes for the $\mathrm{Pr}^{3+}$ crystal field at all temperatures. A similar mechanism could explain the broadening of the $\mathrm{Pr}^{3+}$ excitations only above $30-50 \mathrm{~K}$ in $\mathrm{Pr}_{2} \mathrm{O}_{2} \mathrm{Fe}_{2} \mathrm{OSe}_{2}$ : gapped magnetic excitations with $\Delta \approx 5 \mathrm{meV} \approx 58 \mathrm{~K}$ mean that such decay channels are only thermally and kinetically accessible at these high temperatures. Our results apply to $\mathrm{Pr}^{3+}$ oxyselenides, but it is also interesting to consider other lanthanide oxyselenides: ${ }^{139} \mathrm{La}$ spin relaxation in $\mathrm{La}_{2} \mathrm{O}_{2} \mathrm{Fe}_{2} \mathrm{OSe}_{2}$ has been investigated by NMR [12], and an anomaly in the relaxation rate was observed at $\sim 50 \mathrm{~K}$, the same temperature below which ligand field excitations sharpen in $\mathrm{Pr}_{2} \mathrm{O}_{2} \mathrm{Fe}_{2} \mathrm{OSe}_{2}$. The temperature dependence of the NMR relaxation rate was analyzed in terms of thermally activated gapped excitations, consistent with the interpretation involving coupled crystalline and transition metal magnetic excitations proposed here.

\section{CONCLUSION}

The crystal and magnetic structures of $\mathrm{Pr}_{2} \mathrm{O}_{2} M_{2} \mathrm{OSe}_{2}(M=$ $\mathrm{Mn}, \mathrm{Fe}$ ) have been explored using neutron scattering experiments in a zero field (summarized in Fig. 12) and applied magnetic field $(M=\mathrm{Fe})$. Whilst the magnetic structures 


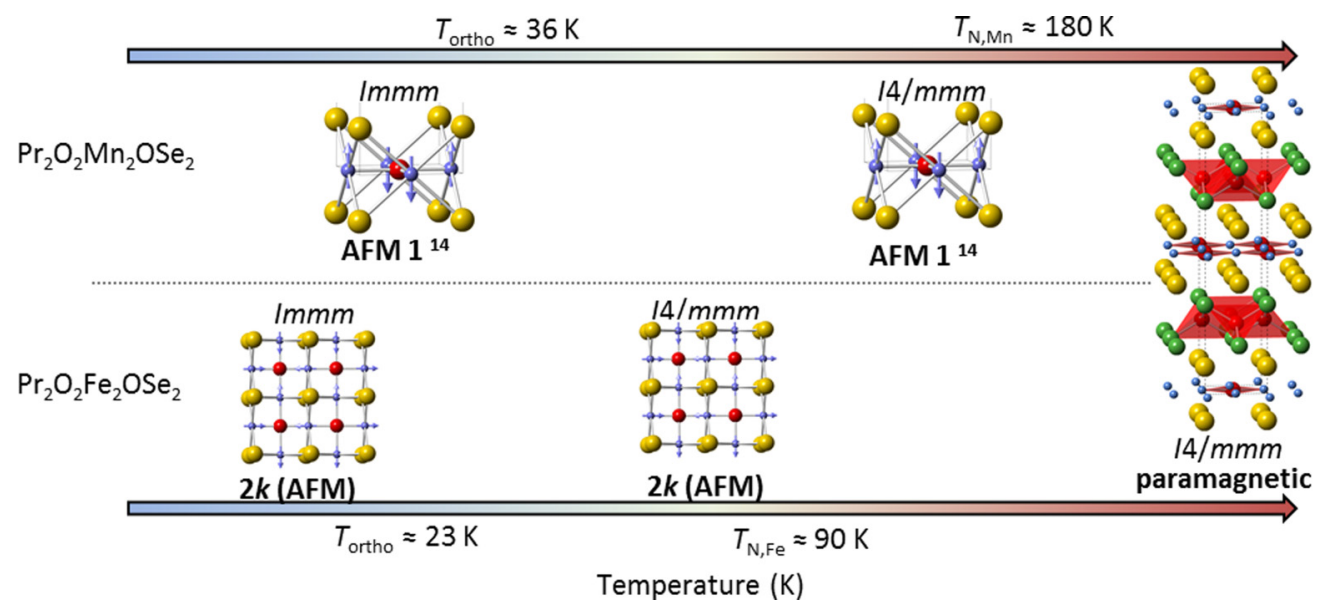

FIG. 12. Schematic illustrating phase transitions of $\operatorname{Pr}_{2} \mathrm{O}_{2} M_{2} \mathrm{OSe}_{2}$ (in zero field) as a function of temperature (temperature axis not to scale), showing the tetragonal paramagnetic phase at high temperatures (right), cooling through $T_{\mathrm{N}}$ to the tetragonal AFM phases (AFM1 for $M=\mathrm{Mn}$ [14], $2 k$ for $M=\mathrm{Fe}$ ), and structural distortion to the orthorhombic Immm phase at low temperatures (left). We note that this orthorhombic distortion is much more subtle for $M=\mathrm{Fe}$ than for $M=\mathrm{Mn}$ and that in zero field, we have no evidence for long-range ordering of $\mathrm{Pr}^{3+}$ moments below this structural transition temperature.

and exchange interactions are similar to those observed for other lanthanide analogues $[10,14,15,17,36,37]$, the structural chemistry is influenced by the symmetry-lowering structural distortion driven by the non-Kramers $\mathrm{Pr}^{3+}$ ion. Analysis of CEFs suggests some coupling between $\mathrm{Pr}^{3+}$ CEFs and transition metal magnetic excitations. This may give some insight into the non-Heisenberg coupling between the lanthanide and transition metal sublattices reported for oxypnictides and oxychalcogenides [12,38]. $\mathrm{Pr}_{2} \mathrm{O}_{2} \mathrm{Fe}_{2} \mathrm{OSe}_{2}$ is more resistant to the $\mathrm{Pr}^{3+}$-driven orthorhombic distortion than the $M=\mathrm{Mn}$ analogue [14], and this resistance is likely to be due to its magnetic behavior. Further INS experiments to study CEFs with temperature in other magnetic systems would add to our understanding of this coupling.

\section{ACKNOWLEDGMENTS}

We are grateful to The Carnegie Trust, Engineering and Physical Sciences Research Council (EPSRC) (Grant No. EP/J011533/1), Science and Technology Facilities Council (STFC), and the University of Kent for funding. We thank the ISIS neutron source for beamtime and Dr. Pascal Manuel and Dr. Laurent Chapon for assistance collecting NPD data.
[1] M. A. Kastner, R. J. Birgeneau, G. Shirane, and Y. Endoh, Rev. Mod. Phys. 70, 897 (1998).

[2] R. J. Birgeneau, C. Stock, J. M. Tranquada, and K. Yamada, J. Phys. Soc. Jpn. 75, 111003 (2006).

[3] P. A. Lee, N. Nagaosa, and X. G. Wen, Rev. Mod. Phys. 78, 17 (2006).

[4] J. Paglione and R. L. Greene, Nature Phys. 6, 645 (2010).

[5] D. C. Johnston, Adv. Phys. 59, 803 (2010).

[6] G. R. Stewart, Rev. Mod. Phys. 83, 1589 (2011).

[7] C. Stock, E. E. Rodriguez, O. Sobolev, J. A. Rodriguez-Rivera, R. A. Ewings, J. W. Taylor, A. D. Christianson, and M. A. Green, Phys. Rev. B 90, 121113(R) (2014).

[8] J. M. Mayer, L. F. Schneemeyer, T. Siegrist, J. V. Waszczak, and B. V. Dover, Angew. Chem. Int. Ed. Engl. 31, 1645 (1992).

[9] J.-X. Zhu, R. Yu, H. Wang, L. L. Zhao, M. D. Jones, J. Dai, E. Abrahams, E. Morosan, M. Fang, and Q. Si, Phys. Rev. Lett. 104, 216405 (2010).

[10] H. Kabbour, E. Janod, B. Corraze, M. Danot, C. Lee, M.-H. Whangbo, and L. Cario, J. Am. Chem. Soc. 130, 8261 (2008).

[11] L. L. Zhao, S. Wu, J. K. Wang, J. P. Hodges, C. Broholm, and E. Morosan, Phys. Rev. B 87, 020406(R) (2013).
[12] M. Gönther, S. Kamusella, R. Sarkar, T. Goltz, H. Luetkens, G. Pascua, S.-H. Do, K.-Y. Choi, H. D. Zhou, C. G. F. Blum, S. Wurmehl, B. Böchner, and H.-H. Klauss, Phys. Rev. B 90, 184408 (2014).

[13] R. H. Liu, J. S. Zhang, P. Cheng, X. G. Luo, J. J. Ying, Y. J. Yan, M. Zhang, A. F. Wang, Z. J. Xiang, G. J. Ye, and X. H. Chen, Phys. Rev. B 83, 174450 (2011).

[14] D. G. Free, N. D. Withers, P. J. Hickey, and J. S. O. Evans, Chem. Mater. 23, 1625 (2011).

[15] N. Ni, E. Climent-Pascual, S. Jia, Q. Huang, and R. J. Cava, Phys. Rev. B 82, 214419 (2010).

[16] D. G. Free and J. S. O. Evans, Phys. Rev. B 81, 214433 (2010).

[17] E. E. McCabe, C. Stock, E. E. Rodriguez, A. S. Wills, J. W. Taylor, and J. S. O. Evans, Phys. Rev. B 89, 100402(R) (2014).

[18] Y. Fuwa, T. Endo, M. Wakeshima, Y. Hinatsu, and K. Ohoyama, J. Am. Chem. Soc. 132, 18020 (2010).

[19] Y. Fuwa, M. Wakeshima, and Y. Hinatsu, Solid State Commun. 150, 1698 (2010).

[20] C. Wang, H.-Q. Tan, C.-M. Feng, Z.-F. Ma, S. Jiang, Z.-A. Xu, G.-H. Cao, K. Matsubayashi, and Y. Uwatoko, J. Am. Chem. Soc. 132, 7069 (2010).

[21] H. Wu, Phys. Rev. B 82, 020410(R) (2010). 
[22] C. Stock and E. E. McCabe, J. Phys.: Condens. Matter 28, 453001 (2016).

[23] E. E. McCabe, D. G. Free, and J. S. O. Evans, Chem. Commun. 47, 1261 (2011).

[24] E. E. McCabe, C. Stock, J. L. Bettis, Jr., M. H. Whangbo, and J. S. O. Evans, Phys. Rev. B 90, 235115 (2014).

[25] Y. Fuwa, M. Wakeshima, and Y. Hinatsu, J. Phys. Condens.: Matter 22, 346003 (2010).

[26] D. Bhoi, P. Mandal, P. Choudhury, S. Pandya, and V. Ganesan, J. Appl. Phys. 110, 113722 (2011).

[27] W. Tian, W. Ratcliff, II, M. G. Kim, J.-Q. Yan, P. A. Kienzle, Q. Huang, B. Jensen, K. W. Dennis, R. W. McCallum, T. A. Lograsso, R. J. McQueeney, A. I. Goldman, J. W. Lynn, and A. Kreyssig, Phys. Rev. B 82, 060514(R) (2010).

[28] G. Hilscher, E. Holland-Moritz, T. Holubar, H.-D. Jostarndt, V. Nekvasil, G. Schaudy, U. Walter, and G. Fillion, Phys. Rev. B 49, 535 (1994).

[29] H.-D. Jostarndt, U. Walter, J. Harnischmacher, J. Kalenborn, A. Severing, and E. Holland-Moritz, Phys. Rev. B 46, 14872 (1992).

[30] L. Soderholme, C.-K. Loong, G. L. Goodman, and B. D. Dabrowski, Phys. Rev. B 43, 7923 (1991).

[31] S. A. J. Kimber, D. N. Argyriou, F. Yokaichiya, K. Habicht, S. Gerischer, T. Hansen, T. Chatterji, R. Klingeler, C. Hess, G. Behr, A. Kondrat, and B. Büchner, Phys. Rev. B 78, 140503(R) (2008).

[32] S. A. J. Kimber, A. H. Hill, Y.-Z. Zhang, H. O. Jeschke, R. Valenti, C. Ritter, I. Schellenberg, W. Hermes, R. Pöttgen, and D. N. Argyriou, Phys. Rev. B 82, 100412(R) (2010).

[33] E. J. Wildman, F. Sher, and A. C. McLaughlin, Inorg. Chem. 54, 2536 (2015).

[34] A. Marcinkova, T. C. Hansen, and J. W. G. Bos, J. Phys.: Condens. Matter 24, 256007 (2012).

[35] A. Marcinkova, T. C. Hansen, C. Curfs, S. Margadonna, and J.-W. G. Bos, Phys. Rev. B 82, 174438 (2010).

[36] N. Ni, S. Jia, Q. Huang, E. Climent-Pascual, and R. J. Cava, Phys. Rev. B 83, 224403 (2011).

[37] E. E. McCabe, A. S. Wills, L. Chapon, P. Manuel, and J. S. O. Evans, Phys. Rev. B 90, 165111 (2014).

[38] H. Maeter, H. Luetkens, Y. G. Pashkevich, A. Kwadrin, R. Khasanov, A. Amato, A. A. Gusev, K. V. Lamonova, D. A. Chervinskii, R. Klingeler, C. Hess, G. Behr, B. Büchner, and H.-H. Klauss, Phys. Rev. B 80, 094524 (2009).

[39] Q. Zhang, W. Tian, H. Li, J.-W. Kim, J. Yan, R. W. McCallum, T. A. Lograsso, J. L. Zarestky, S. L. Bud'ko, R. J. McQueeney, and D. Vaknin, Phys. Rev. B 88, 174517 (2013).

[40] C. Lee, E. Kan, H. Xiang, R. K. Kremer, S.-H. Lee, Z. Hiroi, and M.-H. Whangbo, Inorg. Chem. 51, 6890 (2012).

[41] S. Chi, D. T. Adroja, T. Guidi, R. Bewley, S. Li, J. Zhao, J. W. Lynn, C. M. Brown, Y. Qiu, G. F. Chen, J. L. Lou, N. L. Wang, and P. Dai, Phys. Rev. Lett. 101, 217002 (2008).

[42] Y. Tsukamoto, Y. Okamoto, K. Matsuhira, M.-H. Whangbo, and Z. Hiroi, J. Phys. Soc. Jpn. 80, 094708 (2011).

[43] O. V. Gornostaeva, K. V. Lamonova, S. M. Orel, and Y. G. Pashkevich, Low Temp. Phys. 39, 343 (2013).

[44] A. Jesche, C. Krellner, M. D. Souza, M. Lang, and C. Geibel, New J. Phys. 11, 103050 (2009).
[45] M. A. McGuire, R. P. Hermann, A. S. Sefat, B. C. Scales, R. Jin, D. Mandrus, F. Grandjean, and G. J. Long, New J. Phys. 11, 025011 (2009).

[46] E. A. Goremychkin, R. Osborn, C. H. Wang, M. D. Lumsden, M. A. McGuire, A. S. Sefat, B. C. Scales, D. Mandrus, H. M. Rønnow, Y. Su, and A. D. Christianson, Phys. Rev. B 83, 212505 (2011).

[47] H. M. Rietveld, J. Appl. Crystallogr. 2, 65 (1969).

[48] A. A. Coelho, TOPAS Academic: General Profile and Structure Analysis Software for Powder Diffraction Data (Bruker AXS, Karlsruhe, Germany, 2007), ed. 4.1.

[49] G. Shirane, S. M. Shapiro, and J. M. Tranquada, Neutron Scattering with a Triple Axis Spectrometer (Cambridge University Press, Cambridge, UK, 2002).

[50] S. Toth and B. Lake, J. Phys.: Condens. Matter 27, 166002 (2015).

[51] See Supplemental Material at http://link.aps.org/supplemental/ 10.1103/PhysRevB.95.174441 for details as highlighted in the text.

[52] B. E. Warren, Phys. Rev. 59, 693 (1941).

[53] J.-H. Her, P. W. Stephens, Y. Gao, G. L. Soloveichik, J. Rijssenbeek, M. Andrus, and J.-C. Zhao, Acta Crystallogr. B 63, 561 (2007).

[54] J. Zhao, Q. Huang, C. de la Cruz, J. W. Lynn, M. D. Lumsden, Z. A. Ren, J. Yang, X. Shen, X. Dong, Z. Zhao, and P. Dai, Phys. Rev. B 78, 132504 (2008).

[55] C. Stock, L. C. Chapon, O. Adamopoulos, A. Lappas, M. Giot, J. W. Taylor, M. A. Green, C. M. Brown, and P. G. Radaelli, Phys. Rev. Lett. 103, 077202 (2009).

[56] T. Hong, M. Kenzelmann, M. M. Turnbull, C. P. Landee, B. D. Lewis, K. P. Schmidt, G. S. Uhrig, Y. Qiu, C. Broholm, and D. Reich, Phys. Rev. B 74, 094434 (2006).

[57] P. C. Hohenberg and W. F. Brinkman, Phys. Rev. B 10, 128 (1974).

[58] H.-J. Koo and M.-H. Whangbo, J. Magn. Magn. Mater. 324, 3859 (2012).

[59] U. Walter, J. Phys. Chem. Solids 45, 401 (1984).

[60] K. C. Turberfield, L. Passell, R. J. Birgeneau, and E. Bucher, J. Appl. Phys. 42, 1746 (1971).

[61] M. T. Hutchings, Solid State Phys. 16, 227 (1964).

[62] R. Sachidanandam, Y. Yildirim, A. B. Harris, A. Aharony, and O. Entin-Wohlman, Phys. Rev. B 56, 260 (1997).

[63] C.-K. Loong and L. Soderholm, Phys. Rev. B 48, 14001 (1993).

[64] A. T. Boothroyd, S. M. Doyle, D. MK. Paul, and R. Osborn, Phys. Rev. B 45, 10075 (1992).

[65] E. A. Goremychkin, R. Osborn, B. D. Rainford, E. D. Bauer, M. B. Maple, and M. Koza, Physica B 378-379, 58 (2006).

[66] P. Urban, D. Davidov, B. Elschner, T. Plefka, and G. Sperlich, Phys. Rev. B 12, 72 (1975).

[67] K. W. Becker, P. Fulde, and J. Keller, Z. Phys. B 28, 9 (1977).

[68] M. Loewenhaupt, B. D. Rainford, and F. Steglich, Phys. Rev. Lett. 42, 1709 (1979).

[69] G. Lang, H.-J. Grafe, D. Paar, F. Hammerath, K. Manthey, G. Behr, J. Werner, and B. Büchner, Phys. Rev. Lett. 104, 097001 (2010).

[70] T. D. Kang, E. Standard, K. H. Ahn, A. A. Sirenko, G. L. Carr, S. Park, Y. J. Choi, M. Ramazanoglu, V. Kiryukhin, and S. W. Cheong, Phys. Rev. B 82, 014414 (2010). 